\title{
P-PARTITIONS AND QUASISYMMETRIC POWER SUMS
}

\author{
RICKY INI LIU AND MICHAEL WESELCOUCH
}

\begin{abstract}
The $(P, \omega)$-partition generating function of a labeled poset $(P, \omega)$ is a quasisymmetric function enumerating certain order-preserving maps from $P$ to $\mathbb{Z}^{+}$. We study the expansion of this generating function in the recently introduced type 1 quasisymmetric power sum basis $\left\{\psi_{\alpha}\right\}$. Using this expansion, we show that connected, naturally labeled posets have irreducible $P$-partition generating functions. We also show that series-parallel posets are uniquely determined by their partition generating functions. We conclude by giving a combinatorial interpretation for the coefficients of the $\psi_{\alpha}$-expansion of the $(P, \omega)$-partition generating function akin to the Murnaghan-Nakayama rule.
\end{abstract}

\section{InTRODUCTION}

For a finite poset $P=(P, \prec)$ with a labeling $\omega: P \rightarrow[n]$, the $(P, \omega)$-partition generating function $K_{(P, \omega)}(\mathbf{x})$ is a quasisymmetric function enumerating certain order-preserving maps from $P$ to $\mathbb{Z}^{+}$. In this paper, we study the expansion of $K_{(P, \omega)}(\mathbf{x})$ in the type 1 quasisymmetric power sum basis $\left\{\psi_{\alpha}\right\}$ introduced by Ballantine, Daugherty, Hicks, Mason, and Niese in [3. Such expansions have previously been considered in the naturally labeled case by Alexandersson and Sulzgruber in [2].

It is well known that the $(P, \omega)$-partition generating function of a disconnected poset is reducible since it can be expressed as a product over the connected components of $P$. McNamara and Ward [14 asked whether the converse is true, namely whether the $(P, \omega)$ partition generating function of a connected poset is always irreducible in QSym. We answer this question affirmatively in the naturally labeled case. In particular, we relate part of the $\psi_{\alpha}$-expansion of $K_{P}(\mathbf{x})$ to certain zigzag labelings of $P$, which exist only for connected posets. We then use this to deduce that connected, naturally labeled posets have irreducible $P$-partition generating functions in Section 4. It remains open whether this is true for all connected labeled posets $(P, \omega)$. (See [10] for some discussion of irreducibility in QSym.)

An important object in this study is a pair of linear functionals $\eta$ and $\tilde{\eta}$ on QSym (defined by the present authors in [11]) that can be used to determine if a poset has exactly 1 minimal or 1 maximal element. These functionals also have the property that they send any reducible element of QSym to 0. In Section 3, we express $\eta$ and $\tilde{\eta}$ in terms of the basis $\left\{\psi_{\alpha}\right\}$ and use this to describe the action of various involutions of QSym on this basis.

In [11], the present authors studied the question of when two distinct (naturally labeled) posets can have the same $P$-partition generating function. This question has been studied extensively in the case of skew Schur functions [4, 13, 15], by McNamara and Ward [14] for general labeled posets, and by Hasebe and Tsujie [8] for rooted trees (and more generally $(N, \bowtie)$-free posets). Hasebe and Tsujie ask in [8] whether series-parallel posets can be distinguished by their partition generating functions. (A poset is series-parallel if it can be built

Date: December 24, 2019.

R. I. Liu and M. Weselcouch were partially supported by National Science Foundation grant DMS-1700302. 
from one-element posets using ordinal sum and disjoint union operations, or alternatively, if it is $N$-free.) We use the previously stated result on irreducibility to give a complete, affirmative answer to this question in Section 5.

Alexandersson and Sulzgruber [2] show that when $(P, \omega)$ is naturally labeled, $K_{P}(\mathbf{x})=$ $\sum_{\alpha} c_{\alpha} \psi_{\alpha}$ is $\psi$-positive, and they give a combinatorial interpretation for the coefficients $c_{\alpha}$. In Section 6, we extend this work and give a (signed) combinatorial interpretation for the coefficients in the $\psi_{\alpha}$-expansion of $K_{(P, \omega)}(\mathbf{x})$ for any labeled poset. This interpretation generalizes the Murnaghan-Nakayama rule for computing the expansion of a skew Schur function in terms of power sum symmetric functions.

In summary, in Section 2 we will give some preliminary information; in Section 3 we describe $\eta, \tilde{\eta}$, and various involutions of QSym in terms of the type 1 quasisymmetric power sum basis $\left\{\psi_{\alpha}\right\}$; in Section 4 we show that connected, naturally labeled posets have irreducible $P$-partition generating functions; in Section 5 we show that series-parallel posets are uniquely determined by their partition generating function; and in Section 6 we give a combinatorial interpretation for the coefficients in the $\psi_{\alpha}$-expansion of $K_{(P, \omega)}(\mathbf{x})$ akin to the Murnaghan-Nakayama rule.

\section{Preliminaries}

We begin with some preliminaries about compositions, posets, quasisymmetric functions, and Hopf algebras. For more information, see [7, 11, 14, 19].

2.1. Compositions and partitions. A composition $\alpha=\left(\alpha_{1}, \alpha_{2}, \ldots, \alpha_{k}\right)$ of $n$ is a finite sequence of positive integers summing to $n$. (When it is clear from context, we will remove the parentheses and commas when writing a composition.) A weak composition of $n$ is a finite sequence of nonnegative integers summing to $n$. A partition of $n$ is a composition of $n$ whose parts are in weakly decreasing order. Given a composition $\alpha$ and partition $\lambda$, we write $\alpha \sim \lambda$ if $\lambda$ is formed by rearranging the parts of $\alpha$ into weakly decreasing order. We use the notation $\alpha \vDash n$ if $\alpha$ is a composition of $n$ and $\lambda \vdash n$ if $\lambda$ is a partition of $n$.

We will use the shorthand $1^{k}$ to denote the composition $(\underbrace{1,1, \ldots, 1}_{k})$. The reverse of $\alpha$, denoted $\alpha^{\text {rev }}$, is the composition formed by reversing the order of $\alpha$. The length of $\alpha$, denoted $l(\alpha)$, is the number of parts of $\alpha$.

The compositions of $n$ are in bijection with the subsets of $[n-1]$ in the following way: for any composition $\alpha$, define

$$
D(\alpha)=\left\{\alpha_{1}, \quad \alpha_{1}+\alpha_{2}, \quad \ldots, \quad \alpha_{1}+\alpha_{2}+\cdots+\alpha_{k-1}\right\} \subseteq[n-1] .
$$

Likewise, for any subset $S=\left\{s_{1}, s_{2}, \ldots, s_{k-1}\right\} \subseteq[n-1]$ with $s_{1}<s_{2}<\cdots<s_{k-1}$, we can define the composition

$$
\operatorname{co}(S)=\left(s_{1}, \quad s_{2}-s_{1}, \quad s_{3}-s_{2}, \quad \ldots, \quad s_{k-1}-s_{k-2}, \quad n-s_{k-1}\right) .
$$

Given two nonempty compositions $\alpha=\left(\alpha_{1}, \alpha_{2}, \ldots, \alpha_{k}\right)$ and $\beta=\left(\beta_{1}, \beta_{2}, \ldots, \beta_{l}\right)$, their concatenation is

$$
\alpha \cdot \beta=\left(\alpha_{1}, \alpha_{2}, \ldots, \alpha_{k}, \beta_{1}, \beta_{2}, \ldots, \beta_{l}\right),
$$

and their near-concatenation is

$$
\alpha \odot \beta=\left(\alpha_{1}, \alpha_{2}, \ldots, \alpha_{k}+\beta_{1}, \beta_{2}, \ldots, \beta_{m}\right) .
$$

Observe that if $\alpha \vDash n$ and $\beta \vDash m$, then both $\alpha \cdot \beta \vDash(n+m)$ and $\alpha \odot \beta \vDash(n+m)$. 
For a partition $\lambda$, define $z_{\lambda}=1^{m_{1}} m_{1} ! \cdot 2^{m_{2}} m_{2} ! \cdots$, with $m_{i}$ being the multiplicity of $i$ in $\lambda$. This number is the size of the centralizer of a group element $g \in S_{n}$ whose cycle type is $\lambda$ [19]. For a composition $\alpha$ where $\alpha \sim \lambda$, we define $z_{\alpha}=z_{\lambda}$.

2.1.1. Refinement. If $\alpha$ and $\beta$ are both compositions of $n$, then we say that $\alpha$ refines $\beta$ (equivalently, $\beta$ coarsens $\alpha$ ), denoted $\alpha \preceq \beta$, if

$$
\beta=\left(\alpha_{1}+\cdots+\alpha_{i_{1}}, \quad \alpha_{i_{1}+1}+\cdots+\alpha_{i_{1}+i_{2}}, \quad \ldots, \quad \alpha_{i_{1}+\cdots+i_{k-1}+1}+\cdots+\alpha_{i_{1}+\cdots+i_{k}}\right),
$$

for some $i_{1}, i_{2}, \ldots, i_{k}$ summing to $l(\alpha)$. Equivalently, $\alpha \preceq \beta$ if and only if $D(\beta) \subseteq D(\alpha)$.

The following function will be important in a number of combinatorial formulas.

Definition 2.1. Given a refinement $\alpha$ of $\beta$, let $\alpha^{(i)}$ be the composition consisting of the parts of $\alpha$ that combine to form $\beta_{i}$, so $\alpha^{(i)} \vDash \beta_{i}$. We then define

$$
\pi(\alpha)=\prod_{i=1}^{l(\alpha)} \sum_{j=1}^{i} \alpha_{j} \quad \text { and } \quad \pi(\alpha, \beta)=\prod_{i=1}^{l(\beta)} \pi\left(\alpha^{(i)}\right) .
$$

Observe that $\pi(\alpha)=\pi(\alpha,(n))$.

Example 2.2. If $\alpha=(1,1,4,2,1)$ and $\beta=(2,7)$, then $\alpha \preceq \beta$, and

$$
\pi(\alpha, \beta)=(1 \cdot(1+1))(4 \cdot(4+2) \cdot(4+2+1))=336 .
$$

This function has the following combinatorial interpretation. Given compositions $\alpha, \beta \vDash n$ with $\alpha \preceq \beta$, let

$$
\begin{array}{ll}
D(\alpha)=\left\{s_{1}, s_{2}, \ldots, s_{l-1}\right\}, & 0=s_{0}<s_{1}<s_{2}<\cdots<s_{l-1}<s_{l}=n, \\
D(\beta)=\left\{s_{i_{1}}, s_{i_{2}}, \ldots, s_{i_{k-1}}\right\}, & 0=i_{0}<i_{1}<i_{2}<\cdots<i_{k-1}<i_{k}=l .
\end{array}
$$

Let $\Pi(\alpha, \beta)$ (denoted Cons $_{\alpha \preceq \beta}$ in [3]) be the set of permutations $\sigma \in S_{n}$ such that $\sigma_{j} \leq \sigma_{s_{i}}$ for all $s_{i_{m-1}}<j \leq s_{i} \leq s_{i_{m}}$, where $1 \leq m \leq k$. Alternatively, these are the permutations that satisfy $\sigma_{s_{i}}=\max _{j \in\left(s_{i_{m-1}}, s_{i}\right]} \sigma_{j}$ whenever $i_{m-1}<i \leq i_{m}$. In other words, suppose that the letters in $\sigma$ are broken into blocks with sizes given by the parts of $\beta$ and then into subblocks with sizes given by the parts of $\alpha$. Then the maximum of any subblock must occur at the end of that subblock, and these maximum entries must increase within any block.

Example 2.3. Let $\alpha=(1,1,4,2,1)$ and $\beta=(2,7)$. It follows that $D(\alpha)=\{1,2,6,8\}$ and $D(\beta)=\{2\}$. Then (using bars to indicate the composition $\beta$ and spaces to indicate the refinement given by $\alpha$ )

$$
\sigma=23 \mid 6417589 \in \Pi(\alpha, \beta)
$$

but

$$
\sigma^{\prime}=14 \mid 2738569 \notin \Pi(\alpha, \beta)
$$

because $\sigma_{8} \neq \max \left\{\sigma_{3}, \ldots, \sigma_{8}\right\}$.

The following result is Lemma 3.7 from [3]; we sketch a proof here for completeness.

Lemma 2.4. For compositions $\alpha \preceq \beta$,

$$
|\Pi(\alpha, \beta)|=\frac{n !}{\pi(\alpha, \beta)} .
$$


Proof. Choose $i_{m-1}<i \leq i_{m}$. For a random permutation $\sigma \in S_{n}$, the probability that $\sigma_{s_{i}}=\max _{j \in\left(s_{i_{m-1}}, s_{i}\right]} \sigma_{j}$ is

$$
\frac{1}{s_{i}-s_{i_{m-1}}}=\frac{1}{\alpha_{1}^{(m)}+\cdots+\alpha_{i-i_{m-1}}^{(m)}} .
$$

It is easy to check that these probabilities are independent, and taking the product over all $i$ gives $\frac{1}{\pi(\alpha, \beta)}$.

Using Lemma 2.4, one can prove the following identity, which we will need later.

Lemma 2.5. Let $\alpha$ be a composition of $n$. Then

$$
\frac{n !}{\pi\left(\alpha^{r e v}\right)}=\sum_{\beta \succeq \alpha}(-1)^{l(\alpha)-l(\beta)} \frac{n !}{\pi(\alpha, \beta)} .
$$

Proof. Let $l(\alpha)=l$ and $D(\alpha)=\left\{s_{1}, s_{2}, \ldots, s_{l-1}\right\}$ with $0=s_{0}<s_{1}<s_{2}<\cdots<s_{l-1}<s_{l}=$ $n$.

By an argument similar to the one used in the proof of Lemma 2.4, the left hand side counts the number of permutations $\sigma$ such that for all $i=1, \ldots, l, \max \left\{\sigma_{s_{i-1}+1}, \ldots, \sigma_{n}\right\}=\sigma_{s_{i}}$. In other words, out of the last $\alpha_{i}+\cdots+\alpha_{l}$ values of $\sigma$, the largest of those values is in position $s_{i}$. Observe that these are the permutations in $\Pi(\alpha, \alpha)$ such that $\sigma_{s_{1}}>\sigma_{s_{2}}>\cdots>\sigma_{s_{l}}$.

The right hand side counts pairs $(\sigma, \beta)$ such that $\sigma \in \Pi(\alpha, \beta) \subseteq \Pi(\alpha, \alpha)$ with a sign depending on the length of $\beta$. We will describe a sign-reversing involution whose fixed points are the permutations that are counted by the left hand side. Let $j$ be the smallest positive number such that $\sigma_{s_{j}}<\sigma_{s_{j+1}}$. Then $\sigma \in \Pi\left(\alpha, \beta^{\prime}\right)$, where

$$
D\left(\beta^{\prime}\right)= \begin{cases}D(\beta) \cup\left\{s_{j}\right\} & \text { if } s_{j} \notin D(\beta), \\ D(\beta) \backslash\left\{s_{j}\right\} & \text { if } s_{j} \in D(\beta) .\end{cases}
$$

In either case, $l\left(\beta^{\prime}\right)=l(\beta) \pm 1$, so $(\sigma, \beta) \mapsto\left(\sigma, \beta^{\prime}\right)$ is a sign-reversing involution. The fixed points are exactly the permutations in $\Pi(\alpha, \alpha)$ where $\sigma_{s_{1}}>\sigma_{s_{2}}>\cdots>\sigma_{s_{l}}$, which are counted by the left hand side.

2.1.2. Shuffles. An important notion when working with compositions and quasisymmetric functions is that of shuffles.

Definition 2.6. Let $\alpha=\left(\alpha_{1}, \alpha_{2}, \ldots, \alpha_{k}\right)$ and $\beta=\left(\beta_{1}, \beta_{2}, \ldots, \beta_{l}\right)$. The multiset of shuffles $\alpha \amalg \beta$ consists of all $\left(\begin{array}{c}k+l \\ k\end{array}\right)$ compositions of length $k+l$ that contain $\alpha$ and $\beta$ as disjoint subsequences, counted with multiplicity.

Example 2.7. Let $\alpha=(4,2,1)$ and $\beta=(3,1)$, then the multiset of shuffles of $\alpha$ and $\beta$ is:

$$
\begin{aligned}
& (4,2,1,3,1),(4,2,3,1,1),(4,2,3,1,1),(4,3,2,1,1),(4,3,2,1,1), \\
& (4,3,1,2,1),(3,4,2,1,1),(3,4,2,1,1),(3,4,1,2,1),(3,1,4,2,1) .
\end{aligned}
$$

The shuffle operator $\amalg$ is commutative, meaning $\alpha \amalg \beta=\beta ш \alpha$ as multisets. 
2.2. Posets and $P$-partitions. Let $P=(P, \prec)$ be a finite poset of size $n$. A labeling of $P$ is a bijection $\omega: P \rightarrow\{1,2, \ldots, n\}$.

Definition 2.8. For a labeled poset $(P, \omega)$, a $(P, \omega)$-partition is a map $\theta: P \rightarrow \mathbb{Z}^{+}$that satisfies the following:

(a) If $x \preceq y$, then $\theta(x) \leq \theta(y)$.

(b) If $x \preceq y$ and $\omega(x)>\omega(y)$, then $\theta(x)<\theta(y)$.

Definition 2.9. The $(P, \omega)$-partition generating function $K_{(P, \omega)}\left(x_{1}, x_{2}, \ldots\right)$ for a labeled poset $(P, \omega)$ is given by

$$
K_{(P, \omega)}(\mathbf{x})=K_{(P, \omega)}\left(x_{1}, x_{2}, \ldots\right)=\sum_{(P, \omega) \text {-partition } \theta} x_{1}^{\left|\theta^{-1}(1)\right|} x_{2}^{\left|\theta^{-1}(2)\right|} \cdots,
$$

where the sum ranges over all $(P, \omega)$-partitions $\theta$.

Note that $K_{(P, \omega)}\left(x_{1}, x_{2}, \ldots\right)$ depends only on the relative order of $\omega(x)$ and $\omega(y)$ when $y$ covers $x$. In the Hasse diagram of $(P, \omega)$, we will use a bold edge (or strict edge) to represent when $x \prec y$ but $\omega(x)>\omega(y)$, while we will use a plain edge (or natural edge) when $x \prec y$ and $\omega(x)<\omega(y)$. We will replace the nodes of a labeled poset by circles with the label inside.

Example 2.10. The following is a depiction of a labeled poset $(P, \omega)$ for $n=3$. Note that there is one strict edge and one natural edge.

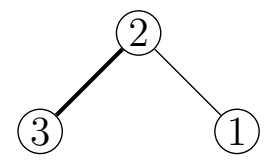

If $\omega$ is order-preserving, then $K_{(P, \omega)}\left(x_{1}, x_{2}, \ldots\right)$ depends only on the structure of $P$. In this case, we call $P$ naturally labeled and write $K_{P}(\mathbf{x})=K_{(P, \omega)}(\mathbf{x})$.

Remark 2.11. If $P$ is a disconnected poset, then $K_{(P, \omega)}(\mathbf{x})$ can be written as a product of $K_{\left(P_{i}, \omega_{i}\right)}(\mathbf{x})$, where the $P_{i}$ are the connected components of $P$ (and $\omega_{i}$ is obtained by appropriately restricting $\omega$ to $P_{i}$ ).

A linear extension of a labeled poset $(P, \omega)$ is a permutation $\pi$ of $[n]$ that respects the relations in $(P, \omega)$, that is, if $x \preceq y$, then $\pi^{-1}(\omega(x)) \leq \pi^{-1}(\omega(y))$. The set of all linear extensions of $(P, \omega)$ is denoted $\mathcal{L}(P, \omega)$. Note that $|\mathcal{L}(P, \omega)|$ is the coefficient of $x_{1} x_{2} \cdots x_{n}$ in $K_{(P, \omega)}(\mathbf{x})$.

2.3. Quasisymmetric Functions. A quasisymmetric function in the variables $x_{1}, x_{2}, \ldots$ (with coefficients in $\mathbb{C}$ ) is a formal power series $f(\mathbf{x}) \in \mathbb{C}[[\mathbf{x}]]$ of bounded degree such that, for any composition $\alpha$, the coefficient of $x_{1}^{\alpha_{1}} x_{2}^{\alpha_{2}} \cdots x_{k}^{\alpha_{k}}$ equals the coefficient of $x_{i_{1}}^{\alpha_{1}} x_{i_{2}}^{\alpha_{2}} \cdots x_{i_{k}}^{\alpha_{k}}$ whenever $i_{1}<i_{2}<\cdots<i_{k}$. We denote the algebra of quasisymmetric functions by QSym $=$ $\bigoplus_{n \geq 0} \mathrm{QSym}_{n}$, graded by degree.

We will consider the following bases for QSym: the monomial basis $\left\{M_{\alpha}\right\}$, the fundamental quasisymmetric function basis $\left\{L_{\alpha}\right\}$, and the (unnormalized) type 1 quasisymmetric power sum basis $\left\{\psi_{\alpha}\right\}$ introduced in [3]. by

The monomial quasisymmetric function basis $\left\{M_{\alpha}\right\}$, indexed by compositions $\alpha$, is given

$$
M_{\alpha}=\sum_{1 \leq i_{1}<i_{2}<\cdots<i_{k}} x_{i_{1}}^{\alpha_{1}} x_{i_{2}}^{\alpha_{2}} \cdots x_{i_{k}}^{\alpha_{k}} .
$$


The fundamental quasisymmetric function basis $\left\{L_{\alpha}\right\}$, also indexed by compositions $\alpha$, is given by

$$
L_{\alpha}=\sum_{\substack{i_{1} \leq \cdots \leq i_{n} \\ i_{s}<i_{s+1} \text { if } s \in D(\alpha)}} x_{i_{1}} x_{i_{2}} \cdots x_{i_{n}} .
$$

In terms of the monomial basis, $L_{\alpha}=\sum_{\beta \preceq \alpha} M_{\beta}$, where the sum runs over all refinements $\beta$ of $\alpha$. By Möbius inversion, this implies that $M_{\alpha}=\sum_{\beta \preceq \alpha}(-1)^{l(\beta)-l(\alpha)} L_{\beta}$.

For any labeled poset $(P, \omega), K_{(P, \omega)}(\mathbf{x})$ is a quasisymmetric function, and we can express it in terms of the fundamental basis $\left\{L_{\alpha}\right\}$ using the linear extensions of $(P, \omega)$. For any linear extension $\pi \in \mathcal{L}(P, \omega)$, define the descent set of $\pi$ to be $\operatorname{des}(\pi)=\{i \mid \pi(i)>\pi(i+1)\}$. Abbreviating $\operatorname{co}(\operatorname{des}(\pi))$ by $\operatorname{co}(\pi)$, we then have the following result.

Theorem $2.12([6,18])$. Let $(P, \omega)$ be a labeled poset. Then

$$
K_{(P, \omega)}(\mathbf{x})=\sum_{\pi \in \mathcal{L}(P, \omega)} L_{\operatorname{co}(\pi)}
$$

2.3.1. Quasisymmetric power sums. Formally, the type 1 quasisymmetric power sum basis, as defined in [3], is the basis $\left\{\Psi_{\alpha}\right\}$ of QSym that satisfies $\left\langle\Psi_{\alpha}, \boldsymbol{\Psi}_{\beta}\right\rangle=z_{\alpha} \delta_{\alpha, \beta}$, where $\boldsymbol{\Psi}_{\beta}$ is the noncommutative power sum of the first kind (introduced in [5]). The type 1 quasisymmetric power sum basis refines the power sum symmetric functions (see Section 6.3) as

$$
p_{\lambda}=\sum_{\alpha \sim \lambda} \Psi_{\alpha}
$$

where the sum runs over all compositions $\alpha$ that rearrange to the partition $\lambda$. We will consider the unnormalized version of the type 1 quasisymmetric power sum basis with basis elements $\left\{\psi_{\alpha}\right\}$ given by $\psi_{\alpha}=\frac{\Psi_{\alpha}}{z_{\alpha}}$. From now on, when we refer to the type 1 quasisymmetric power sum basis, we are referring to the unnormalized version $\psi_{\alpha}$ unless stated otherwise.

We can express $\psi_{\alpha}$ in terms of the monomial basis:

$$
\psi_{\alpha}=\sum_{\beta \succeq \alpha} \frac{1}{\pi(\alpha, \beta)} M_{\beta},
$$

where the sum runs over all coarsenings $\beta$ of $\alpha$. This was proven in [3], but for the purposes of this paper, this can be taken as the definition of $\psi_{\alpha}$.

For more information on the type 1 quasisymmetric power sum basis, see [2, 3].

2.3.2. Length. The type 1 quasisymmetric power sum basis has the following multiplicative property as shown in [3]:

$$
\psi_{\alpha} \psi_{\beta}=\sum_{\gamma \in \alpha \amalg \beta} \psi_{\gamma} .
$$

Every composition $\gamma$ in the $\psi$-support of $\psi_{\alpha} \psi_{\beta}$ satisfies $l(\gamma)=l(\alpha)+l(\beta)$. We can then use the $\psi$-basis to refine $\mathrm{QSym}_{n}$ :

$$
\mathrm{QSym}_{n}=\bigoplus_{\lambda \vdash n} \mathrm{QSym}_{\lambda},
$$

where QSym ${ }_{\lambda}$ is spanned by $\left\{\psi_{\alpha}: \alpha \sim \lambda\right\}$. Observe that if $f \in \operatorname{QSym}_{\lambda}$ and $g \in \operatorname{QSym}_{\mu}$, then $f \cdot g \in \mathrm{QSym}_{\nu}$, where $\nu$ is the partition formed by combining and rearranging the parts of $\lambda$ and $\mu$. 
We define QSym $_{n, m}$ to be

$$
\operatorname{QSym}_{n, m}:=\bigoplus_{\substack{\lambda \vdash n \\ l(\lambda)=m}} \text { QSym }_{\lambda} .
$$

If $f \in \mathrm{QSym}_{n, m}$, then we say the length of $f$ is $m$. This gives a grading for $\mathrm{QSym}_{n}$,

$$
\operatorname{QSym}_{n}=\bigoplus_{m \geq 0} \operatorname{QSym}_{n, m} .
$$

If $f \in \operatorname{QSym}_{n_{1}, m_{1}}$ and $g \in \mathrm{QSym}_{n_{2}, m_{2}}$, then $f \cdot g \in \mathrm{QSym}_{n_{1}+n_{2}, m_{1}+m_{2}}$.

2.3.3. Hopf Algebra. The ring of quasisymmetric functions is a Hopf algebra; in particular, it is equipped with a coproduct. For more details on combinatorial Hopf algebras, see [1, 7, 17].

The coproduct is defined on the fundamental quasisymmetric function basis (see Proposition 5.2 .15 in [7]) by

$$
\Delta\left(L_{\alpha}\right):=\sum_{\substack{(\beta, \gamma) \\ \alpha=\beta \cdot \gamma \text { or } \beta \odot \gamma}} L_{\beta} \otimes L_{\gamma} .
$$

The coproduct on the type 1 quasisymmetric power sum basis satisfies

$$
\Delta\left(\psi_{\alpha}\right)=\sum_{\beta \cdot \gamma=\alpha} \psi_{\beta} \otimes \psi_{\gamma}
$$

This follows from the definition of the $\psi_{\alpha}$ in [3]: the $\psi_{\alpha}$ are defined to be dual to the noncommutative power sums $\boldsymbol{\Psi}_{\alpha}$, whose multiplication is given by concatenation of compositions.

We define the graded comultiplication $\Delta_{\alpha}\left(\psi_{\beta}\right)$ to be

$$
\Delta_{\alpha}\left(\psi_{\beta}\right):=\sum_{\substack{\gamma^{(1) \ldots \gamma^{(l)}=\beta} \\ \gamma^{(i) \models \alpha_{i}}}} \psi_{\gamma^{(1)}} \otimes \cdots \otimes \psi_{\gamma^{(l)}},
$$

where each $\gamma^{(i)}$ is a composition of $\alpha_{i}$. (Therefore $\Delta_{\alpha}\left(\psi_{\beta}\right)=0$ unless $\alpha \succeq \beta$.) In other words, the graded coproduct gives one graded component of the iterated coproduct. We define the graded comultiplication on the fundamental quasisymmetric function basis similarly.

From Theorem 2.12 and the coproduct on the fundamental basis described above, one can derive the following comultiplication formula for a $(P, \omega)$-partition generating function:

$$
\Delta\left(K_{(P, \omega)}(\mathbf{x})\right)=\sum_{I} K_{(I, \omega)}(\mathbf{x}) \otimes K_{(P \backslash I, \omega)}(\mathbf{x}),
$$

where $I$ is an order ideal of $P$ (we abuse notation slightly by writing $\omega$ for the appropriate restriction of $\omega$ to either $I$ or $P \backslash I$ ). Iterating and applying the graded comultiplication then gives

$$
\Delta_{\alpha}\left(K_{(P, \omega)}(\mathbf{x})\right)=\sum K_{\left(P_{1}, \omega\right)}(\mathbf{x}) \otimes \cdots \otimes K_{\left(P_{l}, \omega\right)}(\mathbf{x})
$$

where for each $i,\left|P_{i}\right|=\alpha_{i} ; P_{1}, P_{2}, \ldots, P_{l}$ partition $P$; and $P_{1} \cup \cdots \cup P_{i}$ is an order ideal of $P$. 


\section{Operations ON QSym}

In this section, we will express some useful linear functionals in terms of the type 1 quasisymmetric power sum basis. We will then see how some well known automorphisms of QSym act on this basis.

3.1. The $\eta$ and $\tilde{\eta}$ functionals. In [11], the present authors showed the existence of linear functionals $\eta$ and $\tilde{\eta}$ on QSym that satisfy

$$
\eta\left(K_{P}(\mathbf{x})\right)= \begin{cases}1 & \text { if } P \text { has exactly } 1 \text { minimal element } \\ 0 & \text { otherwise }\end{cases}
$$

and

$$
\tilde{\eta}\left(K_{P}(\mathbf{x})\right)= \begin{cases}1 & \text { if } P \text { has exactly } 1 \text { maximal element } \\ 0 & \text { otherwise }\end{cases}
$$

whenever $P$ is a naturally labeled poset. (These functions were denoted $\min _{1}$ and $\max _{1}$ in [11.) On the fundamental quasisymmetric function basis, these functions act as follows:

$$
\eta\left(L_{\alpha}\right)= \begin{cases}(-1)^{k} & \text { if } \alpha=\left(1^{k}, n-k\right) \text { for } 0 \leq k<n \\ 0 & \text { otherwise }\end{cases}
$$

and

$$
\tilde{\eta}\left(L_{\alpha}\right)= \begin{cases}(-1)^{k} & \text { if } \alpha=\left(n-k, 1^{k}\right) \text { for } 0 \leq k<n, \\ 0 & \text { otherwise }\end{cases}
$$

These functions can do more than just determine if a poset has exactly one minimal element or one maximal element: they can be used to test if a quasisymmetric function is irreducible.

Lemma 3.1. For all non-constant, homogeneous $f, g \in \mathrm{QSym}, \eta(f \cdot g)=\tilde{\eta}(f \cdot g)=0$.

Proof. The P-partition generating functions of naturally labeled posets span QSym [20]. Therefore we can express $f$ and $g$ as a linear combination of these partition generating functions. The product of the partition generating functions of any two naturally labeled posets gets sent to 0 by $\eta$ since no disconnected poset has exactly 1 minimal element.

A similar proof shows that $\tilde{\eta}(f \cdot g)=0$.

(One can also easily prove this result using the fundamental basis.)

Note that if $f$ is a (non-constant, homogeneous) quasisymmetric function and $\tilde{\eta}(f) \neq 0$ (or similarly if $\eta(f) \neq 0$ ), then Lemma 3.1 tells us that $f$ is irreducible (and in fact does not lie in the span of homogeneous reducible elements of QSym).

It is straightforward to evaluate $\eta$ and $\tilde{\eta}$ on the monomial basis.

Lemma 3.2. On the monomial basis $\left\{M_{\alpha}\right\}$,

$$
\tilde{\eta}\left(M_{\alpha}\right)=(-1)^{l(\alpha)-1} \alpha_{1} \quad \text { and } \quad \eta\left(M_{\alpha}\right)=(-1)^{l(\alpha)-1} \alpha_{l(\alpha)} .
$$

Proof. Expanding $M_{\alpha}$ in the $\left\{L_{\alpha}\right\}$ basis and applying $\tilde{\eta}$ gives

$$
\tilde{\eta}\left(M_{\alpha}\right)=\sum_{\beta \preceq \alpha}(-1)^{l(\beta)-l(\alpha)} \tilde{\eta}\left(L_{\beta}\right) .
$$


Since $\tilde{\eta}\left(L_{\beta}\right)=0$ unless $\beta=\left(n-k, 1^{k}\right)$ we have

$$
\tilde{\eta}\left(M_{\alpha}\right)=\sum_{i=0}^{\alpha_{1}-1}(-1)^{n-\alpha_{1}+i+1-l(\alpha)} \tilde{\eta}\left(L_{\left(\alpha_{1}-i, 1^{n-\alpha_{1}+i}\right)}\right) .
$$

But $\tilde{\eta}\left(L_{\left(\alpha_{1}-i, 1^{n-\alpha_{1}+i}\right)}\right)=(-1)^{n-\alpha_{1}+i}$, therefore $\tilde{\eta}\left(M_{\alpha}\right)=(-1)^{l(\alpha)-1} \alpha_{1}$ as desired.

A similar argument shows that $\eta\left(M_{\alpha}\right)=(-1)^{l(\alpha)-1} \alpha_{l(\alpha)}$.

We can now evaluate the functions $\tilde{\eta}$ and $\eta$ on the type 1 quasisymmetric power sum basis.

Lemma 3.3. On the type 1 quasisymmetric power sum basis $\left\{\psi_{\alpha}\right\}$,

$$
\tilde{\eta}\left(\psi_{\alpha}\right)=\sum_{i=1}^{l(\alpha)} \frac{(-1)^{l(\alpha)-i}}{\pi\left(\alpha_{1} \cdots \alpha_{i-1}\right) \cdot \pi\left(\left(\alpha_{i+1} \cdots \alpha_{l(\alpha)}\right)^{r e v}\right)},
$$

and

$$
\eta\left(\psi_{\alpha}\right)= \begin{cases}1 & \text { if } \alpha=(n) \\ 0 & \text { otherwise }\end{cases}
$$

Proof. We first calculate $\tilde{\eta}\left(\psi_{\alpha}\right)$. Expanding $\psi_{\alpha}$ in the monomial basis and applying $\tilde{\eta}$ using Lemma 3.2 gives

$$
\tilde{\eta}\left(\psi_{\alpha}\right)=\sum_{\beta \succeq \alpha} \frac{1}{\pi(\alpha, \beta)} \tilde{\eta}\left(M_{\beta}\right)=\sum_{\beta \succeq \alpha} \frac{(-1)^{l(\beta)-1} \beta_{1}}{\pi(\alpha, \beta)} .
$$

Let $l(\alpha)=l$. For each $\beta$ that coarsens $\alpha, \beta_{1}=\alpha_{1}+\cdots+\alpha_{i}$ for some value of $i$, and the composition $\gamma=\left(\beta_{2}, \ldots, \beta_{l(\beta)}\right)$ coarsens $\alpha^{i}:=\left(\alpha_{i+1}, \ldots, \alpha_{l}\right)$. We can then group together the compositions $\beta$ by their first component:

$$
\begin{aligned}
\tilde{\eta}\left(\psi_{\alpha}\right) & =\sum_{i=1}^{l} \sum_{\gamma \succeq \alpha^{i}} \frac{(-1)^{l(\gamma)}\left(\alpha_{1}+\cdots+\alpha_{i}\right)}{\pi\left(\alpha,\left(\alpha_{1}+\cdots+\alpha_{i}\right) \cdot \gamma\right)} \\
& =\sum_{i=1}^{l} \sum_{\gamma \succeq \alpha^{i}} \frac{(-1)^{l(\gamma)}}{\pi\left(\alpha_{1} \cdots \alpha_{i-1}\right) \cdot \pi\left(\alpha^{i}, \gamma\right)} .
\end{aligned}
$$

By Lemma 2.5, we have that, for all $i$,

$$
\begin{aligned}
\sum_{\gamma \succeq \alpha^{i}} \frac{(-1)^{l(\gamma)}}{\pi\left(\alpha_{1} \cdots \alpha_{i-1}\right) \cdot \pi\left(\alpha^{i}, \gamma\right)} & =\frac{(-1)^{l\left(\alpha^{i}\right)}}{\pi\left(\alpha_{1} \cdots \alpha_{i-1}\right)} \sum_{\gamma \succeq \alpha^{i}} \frac{(-1)^{l\left(\alpha^{i}\right)-l(\gamma)}}{\pi\left(\alpha^{i}, \gamma\right)} \\
& =\frac{(-1)^{l-i}}{\pi\left(\alpha_{1} \cdots \alpha_{i-1}\right) \cdot \pi\left(\left(\alpha_{i+1} \cdots \alpha_{l}\right)^{\mathrm{rev}}\right)}
\end{aligned}
$$

as desired.

We will now show that $\eta\left(\psi_{\alpha}\right)=0$ unless $\alpha=(n)$, in which case $\eta\left(\psi_{(n)}\right)=1$. As before, we will express $\psi_{\alpha}$ in terms of the monomial basis, and evaluate $\eta$ on both sides. This gives

$$
\eta\left(\psi_{\alpha}\right)=\sum_{\beta \succeq \alpha} \frac{1}{\pi(\alpha, \beta)} \eta\left(M_{\beta}\right)=\sum_{\beta \succeq \alpha} \frac{(-1)^{l(\beta)-1} \beta_{l(\beta)}}{\pi(\alpha, \beta)} .
$$


When $l(\alpha)>1$, for each $\gamma$ that coarsens $\left(\alpha_{1}, \ldots, \alpha_{l-1}\right)$, both $\beta=\gamma \cdot\left(\alpha_{l}\right)$ and $\beta^{\prime}=\gamma \odot\left(\alpha_{l}\right)$ are coarsenings of $\alpha$. Therefore

$$
\sum_{\beta \succeq \alpha} \frac{(-1)^{l(\beta)-1} \beta_{l(\beta)}}{\pi(\alpha, \beta)}=\sum_{\gamma \succeq\left(\alpha_{1}, \ldots, \alpha_{l-1}\right)}\left(\frac{(-1)^{l(\gamma)} \alpha_{l}}{\pi\left(\alpha, \gamma \cdot\left(\alpha_{l}\right)\right)}+\frac{(-1)^{l(\gamma)-1}\left(\gamma_{l(\gamma)}+\alpha_{l}\right)}{\pi\left(\alpha, \gamma \odot\left(\alpha_{l}\right)\right)}\right) .
$$

But for all $\gamma$,

$$
\pi\left(\alpha, \gamma \cdot\left(\alpha_{l}\right)\right)=\pi\left(\left(\alpha_{1}, \ldots, \alpha_{l-1}\right), \gamma\right) \cdot \alpha_{l}
$$

and

$$
\pi\left(\alpha, \gamma \odot\left(\alpha_{l}\right)\right)=\pi\left(\left(\alpha_{1}, \ldots \alpha_{l-1}\right), \gamma\right) \cdot\left(\gamma_{l(\gamma)}+\alpha_{l}\right)
$$

Therefore each term in the sum vanishes, so $\eta\left(\psi_{\alpha}\right)=0$ when $l(\alpha)>1$. When $\alpha=(n)$, we have

$$
\eta\left(\psi_{(n)}\right)=\frac{n}{\pi((n),(n))}=1
$$

Example 3.4. Let $\alpha=3421$. We have

$$
\tilde{\eta}\left(\psi_{3421}\right)=\frac{(-1)^{3}}{(1 \cdot 3 \cdot 7)}+\frac{(-1)^{2}}{(3)(1 \cdot 3)}+\frac{(-1)^{1}}{(3 \cdot 7)(1)}+\frac{(-1)^{0}}{(3 \cdot 7 \cdot 9)}=\frac{4}{189} .
$$

Due to the simplicity of the behavior of $\eta$ on $\psi_{\alpha}$, we can now compute the coefficients in the $\psi_{\alpha}$-expansion of any quasisymmetric function.

Theorem 3.5. Suppose $f \in \mathrm{QSym}$ and $f=\sum_{\alpha} c_{\alpha} \psi_{\alpha}$. Then

$$
c_{\alpha}=\eta^{\otimes l(\alpha)}\left(\Delta_{\alpha} f\right) \text {. }
$$

Proof. Since $\eta$ is linear, we can prove this by showing that $\eta^{\otimes l(\alpha)}\left(\Delta_{\alpha} \psi_{\beta}\right)=\delta_{\alpha, \beta}$. Recall that

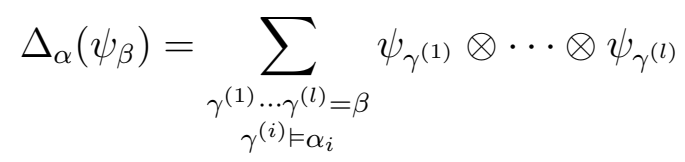

and $\eta\left(\psi_{\alpha}\right)=\delta_{l(\alpha), 1}$. The only way compositions with length 1 can concatenate to $\beta$ is if the compositions are $\left(\beta_{1}\right),\left(\beta_{2}\right), \ldots$. Since then $\beta_{i}=\alpha_{i}$ for all $i$, it follows that $\eta^{\otimes l(\alpha)}\left(\Delta_{\alpha} \psi_{\beta}\right)=$ $\delta_{\alpha, \beta}$.

It should be noted that Theorem 3.5 follows immediately from the combinatorial description of the coefficient of $\psi_{\alpha}$ in $K_{P}(\mathbf{x})$ given by Alexandersson and Sulzgruber [2]. Indeed, consider the following definition of a pointed $P$-partition.

Definition 3.6. Let $P$ be a naturally labeled poset. A $P$-partition $\theta$ is pointed if $\theta$ is surjective onto $[k]$ for some $k$, and $\theta^{-1}(i)$ has a unique minimal element for all $i \in[k]$.

We say that the weight of a pointed $(P, \omega)$-partition $\theta$ is the composition

$$
\operatorname{wt}(\theta)=\left(\left|\theta^{-1}(1)\right|,\left|\theta^{-1}(2)\right|, \ldots\right) .
$$

It is shown in 2] that the coefficient of $\psi_{\alpha}$ in $K_{P}(\mathbf{x})$ is the number of pointed $P$-partitions with weight $\alpha$. This is the same as evaluating $\eta$ on each factor in the graded coproduct of $K_{P}(\mathbf{x})$. We will state this as a corollary to Theorem 3.5. 
Corollary 3.7 ([2], Theorem 5.4). Let $P$ be a naturally labeled poset. Then

$$
K_{P}(\mathbf{x})=\sum_{\theta} \psi_{\mathrm{wt}(\theta)},
$$

where the sum runs over all pointed P-partitions $\theta$.

Proof. Suppose $K_{P}(\mathbf{x})=\sum_{\alpha} c_{\alpha} \psi_{\alpha}$. By Theorem 3.5, $c_{\alpha}=\eta^{\otimes l(\alpha)}\left(\Delta_{\alpha} K_{P}(\mathbf{x})\right)$. Recall that $\eta\left(K_{P}(\mathbf{x})\right)=1$ if $P$ has exactly one minimal element and $\eta\left(K_{P}(\mathbf{x})\right)=0$ otherwise. From equation (囷) above, this means that $\eta^{\otimes l(\alpha)}\left(\Delta_{\alpha} K_{P}(\mathbf{x})\right)$ is the number of ways to partition $P$ into $P_{1}, P_{2}, \ldots, P_{l(\alpha)}$ where for all $i,\left|P_{i}\right|=\alpha_{i}, P_{1} \cup \cdots \cup P_{i}$ is an order ideal of $P$, and $P_{i}$ has exactly one minimal element. This is exactly the number of pointed $P$-partitions with weight $\alpha$.

In Section 6 we will extend this result to all labeled posets.

3.2. Automorphisms. For this section, suppose that $(P, w)$ is a labeled poset. Let $P^{*}$ be the poset formed by reversing the relations of $P$, that is $x \preceq_{P} y \Longleftrightarrow y \preceq_{P^{*}} x$. Let $w^{*}$ be the labeling of $P$ or $P^{*}$ defined by $w^{*}(x)=n-w(x)+1$.

We will consider three well-known automorphisms of QSym: $\omega, \rho$, and their composition $\omega \rho$. (In [12], $\omega \rho$ is denoted by $\psi$.) These automorphisms act as follows on the fundamental basis $\left\{L_{\alpha}\right\}$ :

$$
\begin{aligned}
\omega\left(L_{\alpha}\right) & =L_{\left(\alpha^{c}\right)^{\mathrm{rev}}}, \\
\rho\left(L_{\alpha}\right) & =L_{\alpha^{\mathrm{rev}}}, \\
\omega \rho\left(L_{\alpha}\right) & =L_{\alpha^{c}} .
\end{aligned}
$$

Here $\alpha^{c}$ is the composition such that $D(\alpha)$ and $D\left(\alpha^{c}\right)$ are complementary subsets of $[n-1]$.

These automorphisms perform the following actions on $K_{(P, \omega)}(\mathbf{x})$ :

$$
\begin{aligned}
\omega\left(K_{(P, w)}(\mathbf{x})\right) & =K_{\left(P^{*}, w\right)}(\mathbf{x}), \\
\rho\left(K_{(P, w)}(\mathbf{x})\right) & =K_{\left(P^{*}, w^{*}\right)}(\mathbf{x}), \\
\omega \rho\left(K_{(P, w)}(\mathbf{x})\right) & =K_{\left(P, w^{*}\right)}(\mathbf{x}) .
\end{aligned}
$$

Informally, in terms of the Hasse diagram of $P, \rho$ flips $P$ upside down, $\omega \rho$ switches natural edges with strict edges, and $\omega$ does both. Each of these automorphism can be expressed as the composition of the other two. For more on these automorphisms, see Section 3.6 in [12].

The authors of [3] show that $\omega\left(\psi_{\alpha}\right)=(-1)^{|\alpha|-l(\alpha)} \psi_{\alpha^{\text {rev }}}$ (though they do not give an expansion for the other two automorphisms). This result is easy to deduce given the earlier results in this section, as we now show.

Theorem 3.8 ([3]). For any composition $\alpha, \omega\left(\psi_{\alpha}\right)=(-1)^{|\alpha|-l(\alpha)} \psi_{\alpha^{\text {rev }}}$.

Proof. If $\alpha=\left(1^{k}, n-k\right)$, then $\left(\alpha^{c}\right)^{\text {rev }}=\left(1^{n-k-1}, k+1\right)$. Hence, for all compositions $\alpha$, $\eta \circ \omega\left(L_{\alpha}\right)=(-1)^{|\alpha|-1} \eta\left(L_{\alpha}\right)$. In other words, $\eta$ ow acts like $(-1)^{n-1} \eta$ on $\operatorname{QSym}_{n}$.

By Theorem 3.5, the coefficient of $\psi_{\beta}$ in $\omega\left(\psi_{\alpha}\right)$ is $\eta^{\otimes l(\beta)} \Delta_{\beta}\left(\omega\left(\psi_{\alpha}\right)\right)$. From the action of $\omega$ on the fundamental basis $L_{\alpha}$ as given above, we see that

$$
\Delta_{\beta} \circ \omega=\operatorname{rev}\left(\omega^{\otimes l(\beta)} \circ \Delta_{\beta^{\mathrm{rev}}}\right),
$$

where rev is the automorphism of $\mathrm{QSym}^{\otimes l(\beta)}$ that reverses tensor factors. Thus since $\eta^{\otimes l(\beta)} \circ$ rev $=\eta^{\otimes l(\beta)}$,

$$
\eta^{\otimes l(\beta)} \Delta_{\beta}\left(\omega\left(\psi_{\alpha}\right)\right)=(\eta \circ \omega)^{\otimes l(\beta)} \Delta_{\beta^{\mathrm{rev}}}\left(\psi_{\alpha}\right) .
$$


This is only nonzero if $\beta=\alpha^{\text {rev }}$, in which case it equals $\prod_{i}(-1)^{\left|\alpha_{i}\right|-1}=(-1)^{|\alpha|-l(\alpha)}$.

Since $\eta\left(L_{\alpha}\right)=\tilde{\eta}\left(L_{\alpha^{\text {rev }}}\right)$, it follows that $\eta \circ \rho=\tilde{\eta}$. This allows us to give an expansion for $\omega \rho\left(\psi_{\alpha}\right)$ in the type 1 quasisymmetric power sum basis.

Theorem 3.9. Let $\rho\left(\psi_{\alpha}\right)=\sum_{\beta} c_{\beta} \psi_{\beta}$. Then

$$
c_{\beta}=\tilde{\eta}^{\otimes l(\beta)} \Delta_{\beta^{\mathrm{rev}}}\left(\psi_{\alpha}\right) .
$$

Proof. Using the fact that $\eta \circ \rho=\tilde{\eta}$, we have from Theorem 3.5 that

$$
c_{\beta}=\eta^{\otimes l(\beta)} \Delta_{\beta}\left(\rho\left(\psi_{\alpha}\right)\right)=(\eta \circ \rho)^{\otimes l(\beta)} \Delta_{\beta^{\mathrm{rev}}}\left(\psi_{\alpha}\right)=\tilde{\eta}^{\otimes l(\beta)} \Delta_{\beta^{\mathrm{rev}}}\left(\psi_{\alpha}\right) .
$$

Here we use the fact that, as with $\omega$ in Theorem $3.8, \Delta_{\beta} \circ \rho=\operatorname{rev}\left(\rho^{\otimes l(\beta)} \circ \Delta_{\beta^{\text {rev }}}\right)$.

Combining these results immediately gives the following theorem.

Theorem 3.10. Let $\omega \rho\left(\psi_{\alpha}\right)=\sum_{\beta} c_{\beta} \psi_{\beta}$. Then

$$
c_{\beta}=(-1)^{|\beta|-l(\beta)} \tilde{\eta}^{\otimes l(\beta)} \Delta_{\beta}\left(\psi_{\alpha}\right) .
$$

In particular, $\psi_{\beta}$ can only appear in the expansion of $\rho\left(\psi_{\alpha}\right)$ if $\beta^{\text {rev }}$ coarsens $\alpha$, and likewise $\psi_{\beta}$ can only appear in the expansion of $\omega \rho\left(\psi_{\alpha}\right)$ if $\beta$ coarsens $\alpha$. Of course, these coefficients can be computed explicitly using Lemma 3.3.

Example 3.11. By the previous theorem, the expansion of $\omega \rho\left(\psi_{3421}\right)$ in the $\psi_{\alpha}$ basis is

$$
\omega \rho\left(\psi_{3421}\right)=\psi_{3421}+\frac{1}{2} \psi_{343}+\frac{1}{4} \psi_{361}+\frac{1}{8} \psi_{37}-\frac{1}{12} \psi_{721}-\frac{1}{24} \psi_{73}-\frac{1}{28} \psi_{91}-\frac{4}{189} \psi_{10} .
$$

\section{Irreducibility of $K_{P}(\mathbf{x})$}

In this section, we will restrict our attention to the case when $P$ is naturally labeled. We will show that in this case, $K_{P}(\mathbf{x})$ is irreducible whenever $P$ is connected, which partially answers a question from [14.

We first give a brief outline of the proof. We begin by showing that $K_{P}(\mathbf{x})$ is irreducible whenever the truncation $\widetilde{K}_{P}(\mathbf{x})$ of $K_{P}(\mathbf{x})$ to its terms of shortest length is irreducible, and we give a combinatorial description of the $\psi_{\alpha}$-expansion of $\widetilde{K}_{P}(\mathbf{x})$. We then use Lemma 3.3 to evaluate $\tilde{\eta}\left(\widetilde{K}_{P}(\mathbf{x})\right)$ as an alternating sum. By interpreting the terms in this sum combinatorially using inclusion-exclusion, we show that $\tilde{\eta}\left(\widetilde{K}_{P}(\mathbf{x})\right)$ counts certain zigzag labelings of $P$ that exist if and only if $P$ is connected. In particular, $\tilde{\eta}\left(\widetilde{K}_{P}(\mathbf{x})\right)>0$ when $P$ is connected, so by Lemma 3.1, we can then conclude our result.

As a remark, it was shown in [10] that a homogeneous quasisymmetric function is reducible in QSym if and only if it is reducible in the ring $\mathbb{C}\left[\left[x_{1}, x_{2}, \ldots\right]\right]$ of formal power series of bounded degree. (Though the authors of [10] work over $\mathbb{Z}$ instead of $\mathbb{C}$, the results of that paper as well as this one hold in either context.) Note also that by standard degree considerations, if a homogeneous quasisymmetric function is reducible, then its irreducible factors must be homogeneous as well. 
4.1. Minimal length. Given a poset $P$ of size $n$, let $m$ be the minimum length of a composition in the $\psi_{\alpha}$-expansion of $K_{P}(\mathbf{x})$. (As we will see shortly, $m$ will equal the number of minimal elements of $P$.) Then define $\widetilde{K}_{P}(\mathbf{x})$ to be the sum of the terms in the $\psi_{\alpha}$-expansion of $K_{P}(\mathbf{x})$ of this minimum length $m$. In other words, $\widetilde{K}_{P}(\mathbf{x})$ is the projection of $K_{P}(\mathbf{x})$ to $\mathrm{QSym}_{n, m}$.

Example 4.1. Let $P$ be the following 5-element poset.

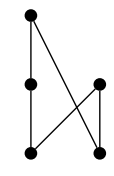

Then

$$
\begin{aligned}
K_{P}(\mathbf{x})= & \psi_{23}+\psi_{14}+2 \psi_{221}+2 \psi_{131}+2 \psi_{122}+\psi_{113} \\
& +2 \psi_{2111}+4 \psi_{1211}+4 \psi_{1121}+2 \psi_{1112}+8 \psi_{11111}, \\
\widetilde{K}_{P}(\mathbf{x})= & \psi_{23}+\psi_{14} .
\end{aligned}
$$

Just as with the usual notion of degree, we can check for irreducibility of a quasisymmetric function by checking irreducibility on the part of minimal length.

Lemma 4.2. If $K_{P}(\mathbf{x})$ is reducible, then $\widetilde{K}_{P}(\mathbf{x})$ is also reducible.

Proof. Recall that multiplication in terms of the type 1 quasisymmetric power sum basis is the shuffle product, so length gives a grading on QSym. If $K_{P}(\mathbf{x})$ is reducible, then $K_{P}(\mathbf{x})$ can be expressed as $K_{P}(\mathbf{x})=f \cdot g$ for some nonconstant homogeneous $f, g \in$ QSym. If $\tilde{f}$ and $\tilde{g}$ are the terms of shortest length in $f$ and $g$, respectively, then $\widetilde{K}_{P}(\mathbf{x})=\tilde{f} \cdot \tilde{g}$.

It follows that if $\widetilde{K}_{P}(\mathbf{x})$ is irreducible, then $K_{P}(\mathbf{x})$ must also be irreducible.

We now give a combinatorial interpretation for $\widetilde{K}_{P}(\mathbf{x})$ in terms of certain special pointed $P$ partitions. Let $\left\{z_{1}, \ldots, z_{m}\right\}$ be the set of minimal elements of $P$. For any subset $\left\{i_{1}, \ldots, i_{l}\right\} \subseteq$ $[m]$, we will denote by $V\left(z_{i_{1}}, \ldots, z_{i_{l}}\right)$ the filter of $P$ whose minimal elements are $\left\{z_{i_{1}}, \ldots, z_{i_{l}}\right\}$.

Definition 4.3. Suppose $P$ has $m$ minimal elements and $\sigma \in S_{m}$. The $\sigma$-partition $\theta_{\sigma}$ is the pointed $P$-partition that sends any element $x \in P$ to the largest number $j$ such that $z_{\sigma_{j}} \preceq x$. In other words, $\theta_{\sigma}^{-1}(i)=V\left(z_{\sigma_{i}}\right) \backslash V\left(z_{\sigma_{i+1}}, \ldots, z_{\sigma_{m}}\right)$ for $i=1, \ldots, m$.

Denote by $\alpha(\sigma)$ the weight of $\theta_{\sigma}$. Explicitly,

$$
\begin{aligned}
\alpha(\sigma)_{i} & =\left|V\left(z_{\sigma_{i}}\right) \backslash V\left(z_{\sigma_{i+1}}, \ldots, z_{\sigma_{m}}\right)\right| \\
& =\left|V\left(z_{\sigma_{i}}, \ldots, z_{\sigma_{m}}\right)\right|-\left|V\left(z_{\sigma_{i+1}}, \ldots, z_{\sigma_{m}}\right)\right| .
\end{aligned}
$$

Example 4.4. Let $P$ be the poset shown below (on the left). On the right is the $\sigma$-partition of $P$ when $\sigma=312$ with weight $\alpha(\sigma)=(1,2,5)$.
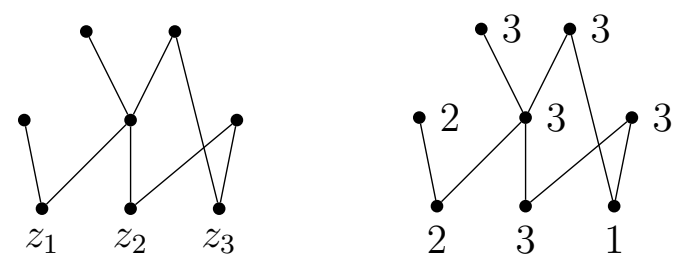
Lemma 4.5. Let $P$ be a poset with $m$ minimal elements. Then

$$
\widetilde{K}_{P}(\mathbf{x})=\sum_{\sigma \in S_{m}} \psi_{\alpha(\sigma)}
$$

Proof. This follows from Corollary 3.7. Note that in any pointed $P$-partition, the minimal elements of $P$ must be sent to different values. Hence the minimum length part of $\widetilde{K}_{P}(\mathbf{x})$ has length (at least) $m$.

If $\theta: P \rightarrow[m]$ is a pointed $P$-partition, then there exists some permutation $\sigma$ such that $\theta\left(z_{\sigma_{i}}\right)=i$ for all $i$. Since $\theta^{-1}(m)$ must be a principal filter of $P$, it must be $V\left(z_{\sigma_{m}}\right)$. Similarly, $\theta^{-1}(m-1)$ must be a principal filter of $P \backslash V\left(z_{\sigma_{m}}\right)$, so it must be $V\left(z_{\sigma_{m-1}}\right) \backslash V\left(z_{\sigma_{m}}\right)$. Continuing in this manner, we see that we must have $\theta=\theta_{\sigma}$, and the result follows.

Example 4.6. Let $P$ be the poset shown in Example 4.4. One can compute for all $\sigma \in S_{3}$ :

$$
\begin{array}{lll}
\alpha(123)=(2,3,3), & \alpha(132)=(2,1,5), & \alpha(213)=(1,4,3), \\
\alpha(231)=(1,2,5), & \alpha(312)=(1,2,5), & \alpha(321)=(1,2,5) .
\end{array}
$$

It follows from Lemma 4.5 that

$$
\widetilde{K}_{P}(\mathbf{x})=\psi_{233}+\psi_{215}+\psi_{143}+3 \psi_{125}
$$

4.2. Evaluation of $\tilde{\eta}$. We will now give a combinatorial interpretation for the value of $\tilde{\eta}$ when applied to some term $\psi_{\alpha(\sigma)}$ appearing in $\widetilde{K}_{P}(\mathbf{x})$. To do this, we will need the following technical notion.

Definition 4.7. Let $\pi, \sigma \in S_{m}$ and let $\left\{z_{1}, \ldots, z_{m}\right\}$ be the set of minimal elements of a poset $P$. We say that a bijection $\phi: P \rightarrow[n]$ is a $(\pi, \sigma)$-labeling if the following are true:

(1) $\phi\left(z_{\pi_{1}}\right)>\phi\left(z_{\pi_{2}}\right)>\cdots>\phi\left(z_{\pi_{m}}\right)$; and

(2) for all $y \in P, \phi(y) \geq \phi(z)$, where $z$ is the minimal element such that $\theta_{\sigma}(z)=\theta_{\sigma}(y)$. Denote by $T_{P}(\pi, \sigma)$ the set of all $(\pi, \sigma)$-labelings of $P$.

In other words, unraveling the definition of $\theta_{\sigma}$ in condition (2), we must have $\phi(y) \geq \phi\left(z_{i}\right)$, where $i$ is the number appearing latest in the word of $\sigma$ such that $y \succeq z_{i}$.

Example 4.8. Shown below are the 1423-partition $\theta$ of a poset $P$ (left), and an example of a $(2314,1423)$-labeling $\phi$ (right). Here $z_{1}, z_{2}, z_{3}, z_{4}$ are taken to be the minimal elements from left to right.
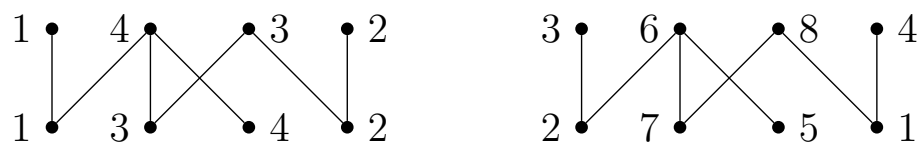

It is clear that condition (1) holds since $\phi\left(z_{2}\right)>\phi\left(z_{3}\right)>\phi\left(z_{1}\right)>\phi\left(z_{4}\right)$.

For condition (2), consider for instance the element $x$ that covers $z_{1}, z_{2}$, and $z_{3}$. Since 3 appears last among 1,2 , and 3 in $\sigma=1423$, it follows that $\theta(x)=\theta\left(z_{3}\right)=\sigma^{-1}(3)=4$, so we must have $\phi(x) \geq \phi\left(z_{3}\right)$.

Consider the operations $f_{i}, g_{i}: S_{m} \rightarrow S_{m}$ for $i=1, \ldots, m-1$ defined by

$$
\begin{aligned}
& f_{i}(\pi)=\pi_{1} \cdots \widehat{\pi}_{i} \cdots \pi_{m} \pi_{i}, \\
& g_{i}(\pi)=\pi_{1} \cdots \pi_{i-1} \pi_{m} \pi_{i} \cdots \pi_{m-1} .
\end{aligned}
$$


Observe that $f_{i}$ and $g_{i}$ are inverses of each other. For any subset $S=\left\{s_{1}, \ldots, s_{l}\right\} \subseteq[m-1]$ with $s_{1}<\cdots<s_{l}$, define

$$
\begin{aligned}
& f_{S}=f_{s_{1}} \circ f_{s_{2}} \circ \cdots \circ f_{s_{l}}, \\
& g_{S}=g_{s_{l}} \circ g_{s_{l-1}} \circ \cdots \circ g_{s_{1}} .
\end{aligned}
$$

Explicitly,

$$
f_{S}(\pi)=\pi_{1} \cdots \widehat{\pi_{s_{1}}} \cdots \widehat{\pi_{s_{2}}} \cdots \widehat{\pi_{s_{l}}} \cdots \pi_{m} \pi_{s_{l}} \pi_{s_{l-1}} \cdots \pi_{s_{1}}
$$

and $g_{S}$ is the inverse of $f_{S}$.

We can now give the following description of $\tilde{\eta}\left(\psi_{\alpha(\sigma)}\right)$.

Lemma 4.9. Let $P$ be a poset of size $n$ with $m$ minimal elements, and let $\sigma \in S_{m}$. Then

$$
(n-1) ! \tilde{\eta}\left(\psi_{\alpha(\sigma)}\right)=\sum_{S \subseteq[m-1]}(-1)^{|S|}\left|T_{P}\left(g_{S}(\sigma), \sigma\right)\right| .
$$

Proof. Note that for fixed $\sigma$, the permutations $g_{S}(\sigma)$ are distinct for distinct sets $S$, so the sets $T_{P}\left(g_{S}(\sigma), \sigma\right)$ are all disjoint.

Fix a value of $i$ with $1 \leq i \leq m$. Every labeling $\phi$ of $P$ in the union

$$
\bigcup_{\substack{S \subseteq[m-1] \\|S|=m-i}} T_{P}\left(g_{S}(\sigma), \sigma\right)
$$

must satisfy the following two conditions:

(1) $\phi\left(z_{\sigma_{1}}\right)>\phi\left(z_{\sigma_{2}}\right)>\cdots>\phi\left(z_{\sigma_{i-1}}\right)>\phi\left(z_{\sigma_{i}}\right)<\phi\left(z_{\sigma_{i+1}}\right)<\cdots<\phi\left(z_{\sigma_{m-1}}\right)<\phi\left(z_{\sigma_{m}}\right)$; and

(2) for all $y \in P, \phi(y) \geq \phi(z)$, where $z$ is the minimal element such that $\theta_{\sigma}(z)=\theta_{\sigma}(y)$.

Equivalently, for all $y \in P, \phi(y)$ must be at least $\phi\left(z_{\sigma_{j}}\right)$ for any $j$ between $i$ and $\theta_{\sigma}(y)$, inclusive. It follows that (1) and (2) are equivalent to the following:

- $\phi\left(z_{\sigma_{i}}\right)=1$ (which of course is the smallest label among all $n$ elements of $P$ );

- for $j=i-1, i-2, \ldots, 1: z_{\sigma_{j}}$ has the smallest label among $P \backslash V\left(z_{\sigma_{j+1}}, \ldots, z_{\sigma_{m}}\right)$, and

- for $j=i+1, i+2, \ldots, m$ : $z_{\sigma_{j}}$ has the smallest label among $V\left(z_{\sigma_{j}}, \ldots, z_{\sigma_{m}}\right)$.

It is easy to check that for a random bijection from $P$ to $[n]$, these events are all independent of one another. If we let $\alpha=\alpha(\sigma)$, then

$$
\begin{aligned}
\left|P \backslash V\left(z_{\sigma_{j+1}}, \ldots, z_{\sigma_{m}}\right)\right| & =\alpha_{1}+\cdots+\alpha_{j}, \\
\left|V\left(z_{\sigma_{j}}, \ldots, z_{\sigma_{m}}\right)\right| & =\alpha_{j}+\cdots+\alpha_{m} .
\end{aligned}
$$

Hence the number of such labelings $\phi$ is

$$
\frac{n !}{n \cdot \prod_{j=1}^{i-1}\left(\alpha_{1}+\cdots+\alpha_{j}\right) \cdot \prod_{j=i+1}^{m}\left(\alpha_{j}+\cdots+\alpha_{m}\right)}=\frac{(n-1) !}{\pi\left(\alpha_{1} \cdots \alpha_{i-1}\right) \cdot \pi\left(\left(\alpha_{i+1} \cdots \alpha_{m}\right)^{\mathrm{rev}}\right)} .
$$

When we include the $(-1)^{|S|}$ and sum over all values of $i$, we find that the right hand side equals

$$
\sum_{i=1}^{m} \frac{(-1)^{m-i}(n-1) !}{\pi\left(\alpha_{1} \cdots \alpha_{i-1}\right) \cdot \pi\left(\left(\alpha_{i+1} \cdots \alpha_{m}\right)^{\mathrm{rev}}\right)}
$$

This value is exactly $(n-1) ! \tilde{\eta}\left(\psi_{\alpha(\sigma)}\right)$ by Lemma 3.3, as desired.

The following two lemmas about $T_{P}(\pi, \sigma)$ will be helpful for giving a combinatorial interpretation for $\tilde{\eta}\left(\widetilde{K}_{P}(\mathbf{x})\right)$. This is because many of the terms on the right hand side of Lemma 4.9 for different $\sigma$ will cancel out due to the principle of inclusion-exclusion. 
Lemma 4.10. Let $P$ be a poset with $m$ minimal elements. For all $S \subseteq[m-1]$ and all $\pi \in S_{m}$,

$$
T_{P}\left(\pi, f_{S}(\pi)\right)=\bigcap_{s \in S} T_{P}\left(\pi, f_{s}(\pi)\right) .
$$

Proof. We will show that the two sets above are equivalent by showing that the inequalities that $\phi$ must satisfy are the same in both sets. Clearly, a labeling $\phi$ must satisfy $\phi\left(z_{\pi_{1}}\right)>$ $\phi\left(z_{\pi_{2}}\right)>\cdots>\phi\left(z_{\pi_{m}}\right)$ to be in either of the sets. Since this determines the inequalities that must be satisfied among minimal elements of $P$, we will turn our attention to some non-minimal element $y \in P$.

Define $S_{y}=\left\{s \in[m] \mid y \succ z_{\pi_{s}}\right\}$. Then the condition on $\phi(y)$ for $\phi \in T_{P}(\pi, \sigma)$ is $\phi(y) \geq \phi\left(z_{\pi_{s}}\right)$, where $\pi_{s}$ is the value that, over all $s \in S_{y}$, appears latest in the word of $\sigma$. Let $a=\max \left(S_{y}\right)=\theta_{\pi}(y)$ (where $\theta_{\pi}$ is the $\pi$-partition of $P$ ), and let $s^{\prime}=\min \left(S_{y} \cap S\right)$ if $S_{y} \cap S \neq \varnothing$. We claim that any labeling $\phi$ in either side of our desired equality must satisfy $\phi(y) \geq \phi\left(z_{\pi_{s^{\prime}}}\right)$ if $S_{y} \cap S \neq \varnothing$, and $\phi(y) \geq \phi\left(z_{\pi_{a}}\right)$ otherwise.

For the left hand side, $f_{S}(\pi)$ by definition reverses the order of $\pi_{s}$ for $s \in S_{y} \cap S$ and places these elements after any element of $S_{y} \backslash S$. Thus the last $\pi_{s}$ for $s \in S_{y}$ appearing in $f_{S}(\pi)$ will be $\pi_{s^{\prime}}$ if $S_{y} \cap S \neq \varnothing$ and $\pi_{a}$ otherwise, as desired.

For the right hand side, a labeling $\phi$ in $T_{P}\left(\pi, f_{s}(\pi)\right)$ must satisfy $\phi(y) \geq \phi\left(z_{\pi_{s}}\right)$ if $s \in S_{y} \cap S$ or $\phi(y) \geq \phi\left(z_{\pi_{a}}\right)$ otherwise. Since we have that $\phi\left(z_{\pi_{1}}\right)>\phi\left(z_{\pi_{2}}\right)>\cdots>\phi\left(z_{\pi_{m}}\right)$ and $s^{\prime}=\min \left(S_{y} \cap S\right)$, it follows that the strictest inequality that $\phi$ must satisfy on the right hand side is $\phi(y) \geq \phi\left(z_{\pi_{s^{\prime}}}\right)$ if $S_{y} \cap S$ is nonempty (when $s=s^{\prime}$ ), and $\phi(y) \geq \phi\left(z_{\pi_{a}}\right)$ otherwise, as desired.

Example 4.11. Consider again the poset shown in Example 4.8, and let $\pi=2314$, so that $\phi\left(z_{2}\right)>\phi\left(z_{3}\right)>\phi\left(z_{1}\right)>\phi\left(z_{4}\right)$.

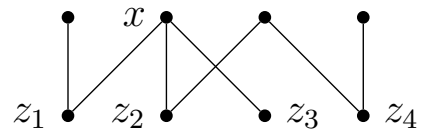

Since the element $x$ covers $z_{1}, z_{2}$, and $z_{3}$, the condition on $\phi(x)$ in $T_{P}(\pi, \sigma)$ depends on which of 1,2 , and 3 appears latest in $\sigma$. For $s=1,2,3$, we then have the following values of $f_{s}(\pi)$ with the corresponding conditions on $\phi(x)$ for each $\phi$ in $T_{P}\left(\pi, f_{s}(\pi)\right)$.

$$
\begin{array}{llrl}
f_{1}(\pi)=3142: & & \phi(x) \geq \phi\left(z_{2}\right), \\
f_{2}(\pi)=2143: & & \phi(x) \geq \phi\left(z_{3}\right), \\
f_{3}(\pi)=2341: & & \phi(x) \geq \phi\left(z_{1}\right) .
\end{array}
$$

Suppose that $S=\{1,2\}$. Then in $\bigcap_{s \in S} T_{P}\left(\pi, f_{s}(\pi)\right)=T_{P}\left(\pi, f_{1}(\pi)\right) \cap T_{P}\left(\pi, f_{2}(\pi)\right)$, we must have $\phi(x) \geq \max \left\{\phi\left(z_{2}\right), \phi\left(z_{3}\right)\right\}=\phi\left(z_{2}\right)$. But this is also the condition imposed on $\phi(x)$ in $T_{P}\left(\pi, f_{S}(\pi)\right)$ since $f_{S}(\pi)=\mathbf{1 4 3 2}$.

We will now see that for all $i$, any $\left(\pi, f_{i}(\pi)\right)$-labeling is also a $(\pi, \pi)$-labeling.

Lemma 4.12. Let $P$ be a poset with $m$ minimal elements. For all $\pi \in S_{m}$,

$$
\bigcup_{i=1}^{m-1} T_{P}\left(\pi, f_{i}(\pi)\right) \subseteq T_{P}(\pi, \pi) .
$$


Proof. Let $\phi \in \bigcup_{i=1}^{m-1} T_{P}\left(\pi, f_{i}(\pi)\right)$. This means that $\phi$ is a $\left(\pi, f_{i}(\pi)\right)$-labeling for some value of $i$. No matter the value of $i, \phi$ must satisfy $\phi\left(z_{\pi_{1}}\right)>\phi\left(z_{\pi_{2}}\right)>\cdots>\phi\left(z_{\pi_{m}}\right)$, which also must be satisfied to be in $T_{P}(\pi, \pi)$.

It remains to show that for all $y \in P, \phi(y) \geq \phi\left(z_{\pi_{a}}\right)$, where $a=\theta_{\pi}(y)$, and $\theta_{\pi}$ is the $\pi$-partition of $P$. If $y \nsucceq z_{\pi_{i}}$, then $\phi(y) \geq \phi\left(z_{\pi_{a}}\right)$. If instead $y \succeq z_{\pi_{i}}$, then $\phi(y) \geq \phi\left(z_{\pi_{i}}\right)$, but $\phi\left(z_{\pi_{i}}\right) \geq \phi\left(z_{\pi_{a}}\right)$ since $i \leq a$ by the definition of $\theta_{\pi}$. Therefore $\phi \in T_{P}(\pi, \pi)$.

As we will show in Lemma 4.16, a $(\pi, \pi)$-labeling $\phi$ appears on the right hand side of Lemma 4.12 but not the left hand side if and only if, for all $i=1, \ldots, m-1$, there exists $y \succ z_{\pi_{i}}$ with $\phi(y)<\phi\left(z_{\pi_{i}}\right)$. We will describe such labelings more thoroughly in the next subsection.

4.3. Zigzag labelings. In this subsection, we introduce the notion of a zigzag labeling, which will allow us to give a combinatorial interpretation for $\tilde{\eta}\left(\widetilde{K}_{P}(\mathbf{x})\right)$.

Definition 4.13. A bijection $\phi: P \rightarrow[n]$ is a zigzag labeling if the following conditions hold:

(1) if $x$ is minimal and $\phi(x) \neq 1$, then there exists $y \succ x$ with $\phi(y)<\phi(x)$; and

(2) if $x$ is not minimal, then there exists a minimal element $y \prec x$ with $\phi(y)<\phi(x)$.

We say that a zigzag labeling $\phi$ has type $\pi$ if $\phi\left(z_{\pi_{1}}\right)>\phi\left(z_{\pi_{2}}\right)>\cdots>\phi\left(z_{\pi_{m}}\right)$.

Example 4.14. The following is an example of a zigzag labeling of $P$ of type 4231. (We take $z_{1}, z_{2}, z_{3}, z_{4}$ to be the minimal elements drawn from left to right.)

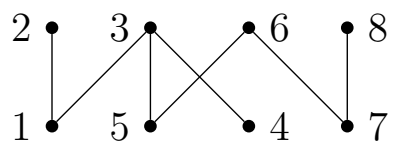

Zigzag labelings can be used to determine whether or not a poset is connected.

Lemma 4.15. A poset $P$ has a zigzag labeling if and only if it is connected.

Proof. Suppose $P$ is connected and has $m$ minimal elements. Since $P$ is connected, there exists a permutation $\pi^{\prime} \in S_{m}$ such that $V\left(z_{\pi_{1}^{\prime}}, \ldots, z_{\pi_{i-1}^{\prime}}\right) \cap V\left(z_{\pi_{i}^{\prime}}\right) \neq \varnothing$ for all $i$. Indeed, if it were not possible to find $\pi_{i}^{\prime}$ given $\pi_{1}^{\prime}, \ldots, \pi_{i-1}^{\prime}$, then $V\left(z_{\pi_{1}^{\prime}}, \ldots, z_{\pi_{i-1}^{\prime}}\right)$ would be a connected component of $P$, which contradicts the fact that $P$ is connected.

An example of a zigzag labeling $\phi$ of $P$ is as follows: label $z_{\pi_{1}^{\prime}}$ with 1 , and label the rest of $V\left(z_{\pi_{1}^{\prime}}\right)$ with $2,3, \ldots$ Then label $z_{\pi_{2}^{\prime}}$ with the lowest number available, and label the elements of $V\left(z_{\pi_{1}^{\prime}}, z_{\pi_{2}^{\prime}}\right) \backslash V\left(z_{\pi_{1}^{\prime}}\right)$ with the next lowest remaining labels. Continue this process until all elements of $P$ are labeled. For all $i>1$, there exists an element $x \in V\left(z_{\pi_{1}^{\prime}}, \ldots, z_{\pi_{i-1}^{\prime}}\right) \cap V\left(z_{\pi_{i}^{\prime}}\right)$, and this element satisfies $x \succ z_{\pi_{i}^{\prime}}$ and $\phi(x)<\phi\left(z_{\pi_{i}^{\prime}}\right)$. Therefore $\phi$ is a zigzag labeling. (One can check that $\phi$ has type $\pi=\left(\pi^{\prime}\right)^{\text {rev }}$.)

Now suppose $P$ is disconnected, and let $\phi: P \rightarrow[n]$ be any bijection. Let $x$ be the element with the smallest label that is not in the same connected component as the element labeled 1. Then $x$ is not comparable to any element $y$ with a smaller label, so $\phi$ cannot be a zigzag labeling.

We will now use the lemmas in the previous subsection to show that we can count zigzag labelings of a fixed type by an alternating sum. 
Lemma 4.16. Let $P$ be a finite poset with $m$ minimal elements, and let $\pi \in S_{m}$. The set of zigzag labelings of $P$ of type $\pi$ is

$$
T_{P}(\pi, \pi) \backslash \bigcup_{i=1}^{m-1} T_{P}\left(\pi, f_{i}(\pi)\right)
$$

and the number of such labelings is

$$
\sum_{S \subseteq[m-1]}(-1)^{|S|}\left|T_{P}\left(\pi, f_{S}(\pi)\right)\right| .
$$

Proof. First note that for a labeling $\phi$ of type $\pi$, condition $(2)$ for being a $(\pi, \pi)$-labeling is equivalent to condition (2) for being a zigzag labeling. Indeed, for any non-minimal $x \in P$, if there exists a minimal element $z_{\pi_{i}} \prec x$ with $\phi\left(z_{\pi_{i}}\right)<\phi(x)$, then certainly we can take $i$ to be the maximum value such that $z_{\pi_{i}} \prec x$ since that will only decrease the value of $\phi\left(z_{\pi_{i}}\right)$.

A labeling $\phi \in T_{P}(\pi, \pi)$ does not lie in $T_{P}\left(\pi, f_{i}(\pi)\right)$ if there exists some element $y \succ z_{\pi_{i}}$ with $\phi(y)<\phi\left(z_{\pi_{i}}\right)$. Hence this occurs for all $i<m$ when $\phi$ satisfies condition (1) of being a zigzag labeling, which completes the proof of the first claim.

From the principle of inclusion-exclusion and Lemma 4.10,

$$
\sum_{S \subseteq[m-1]}(-1)^{|S|}\left|T_{P}\left(\pi, f_{S}(\pi)\right)\right|=\left|T_{P}(\pi, \pi)\right|-\left|\bigcup_{i=1}^{m-1} T_{P}\left(\pi, f_{i}(\pi)\right)\right| .
$$

By Lemma 4.12, we have that

$$
\left|T_{P}(\pi, \pi)\right|-\left|\bigcup_{i=1}^{m-1} T_{P}\left(\pi, f_{i}(\pi)\right)\right|=\left|T_{P}(\pi, \pi) \backslash \bigcup_{i=1}^{m-1} T_{P}\left(\pi, f_{i}(\pi)\right)\right| .
$$

This is exactly the number of zigzag labelings of type $\pi$.

We now have the required tools to prove that the number of zigzag labelings of $P$ can be determined from $K_{P}(\mathbf{x})$.

Theorem 4.17. The number of zigzag labelings of $P$ is counted by $(n-1) ! \tilde{\eta}\left(\widetilde{K}_{P}(\mathbf{x})\right)$.

Proof. By Lemmas 4.5 and 4.9 ,

$$
\begin{aligned}
(n-1) ! \tilde{\eta}\left(\widetilde{K}_{P}(\mathbf{x})\right) & =(n-1) ! \sum_{\sigma \in S_{m}} \tilde{\eta}\left(\psi_{\alpha(\sigma)}\right) \\
& =\sum_{\sigma \in S_{m}} \sum_{S \subseteq[m-1]}(-1)^{|S|}\left|T_{P}\left(g_{S}(\sigma), \sigma\right)\right|
\end{aligned}
$$

For all $\pi \in S_{m}$, we can group together the terms for which $g_{S}(\sigma)=\pi$, meaning $\sigma=f_{S}(\pi)$ since $g_{S}$ and $f_{S}$ are inverses. It follows that

$$
\sum_{\sigma \in S_{m}} \sum_{S \subseteq[m-1]}(-1)^{|S|}\left|T_{P}\left(g_{S}(\sigma), \sigma\right)\right|=\sum_{\pi \in S_{m}} \sum_{S \subseteq[m-1]}(-1)^{|S|}\left|T_{P}\left(\pi, f_{S}(\pi)\right)\right| .
$$

By Lemma 4.16, this counts the number of zigzag labelings of $P$ (of any type).

Example 4.18. Let $P$ be the following naturally labeled poset.

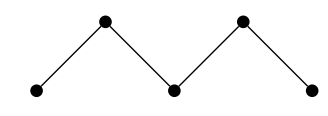


One can compute

$$
K_{P}(\mathbf{x})=4 \psi_{122}+2 \psi_{113}+4 \psi_{1211}+8 \psi_{1121}+4 \psi_{1112}+16 \psi_{11111} .
$$

It follows that

$$
\widetilde{K}_{P}(\mathbf{x})=4 \psi_{122}+2 \psi_{113}
$$

Applying $\tilde{\eta}$ and multiplying by 4 !, we have

$$
4 ! \cdot \tilde{\eta}\left(\widetilde{K}_{P}(\mathbf{x})\right)=24\left(-\frac{1}{6}+\frac{1}{2}\right)=8 .
$$

The following are the 8 zigzag labelings of $P$ :
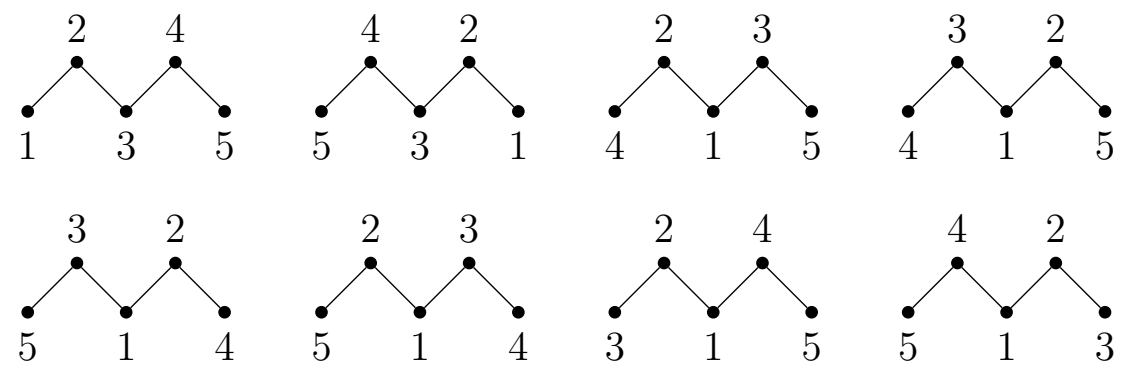

Now that we have this combinatorial interpretation, it is easy to show our main result about the irreduciblity of $K_{P}(\mathbf{x})$.

Theorem 4.19. A poset $P$ is connected if and only if $K_{P}(\mathbf{x})$ is irreducible over QSym.

Proof. The "if" direction is true by Remark 2.11. The "only if" direction is an immediate consequence of Theorem 4.17 and Lemma 4.15. If $P$ is connected, then $(n-1) ! \tilde{\eta}\left(\widetilde{K}_{P}(\mathbf{x})\right)>0$, so it follows from Lemma 3.1 that $\widetilde{K}_{P}(\mathbf{x})$ is irreducible. Therefore by Lemma $4.2, K_{P}(\mathbf{x})$ is irreducible.

In fact, this method implies that if $P$ is connected, then $K_{P}(\mathbf{x})$ does not even lie in the span of the homogeneous, reducible elements of QSym.

This result also tells us how $K_{P}(\mathbf{x})$ factors as a product of irreducible partition generating functions. It is well known that QSym is isomorphic to a polynomial ring and hence is a unique factorization domain (see [9, 10]).

Corollary 4.20. Let $P$ be a naturally labeled poset. Then the irreducible factorization of $K_{P}(\mathbf{x})$ is given by $K_{P}(\mathbf{x})=\prod_{i} K_{P_{i}}(\mathbf{x})$, where $P_{i}$ are the connected components of $P$.

Proof. This follows from the fact that $K_{P}(\mathbf{x})$ factors into a product of the partition generation functions of its connected components, and each of these is irreducible by Theorem 4.19.

This result also gives a condition on when two posets can have the same partition generating function based on their connected components.

Corollary 4.21. Let $P$ and $Q$ be naturally labeled posets. Let $P_{1}, \ldots, P_{k}$ be the connected components of $P$, and $Q_{1}, \ldots, Q_{l}$ the connected components of $Q$. If $K_{P}(\mathbf{x})=K_{Q}(\mathbf{x})$, then $k=l$, and there exists a permutation $\pi \in S_{k}$ such that $K_{P_{i}}(\mathbf{x})=K_{Q_{\pi_{i}}}(\mathbf{x})$ for all $i$.

Proof. This follows immediately from Corollary 4.20 and the fact that QSym is a unique factorization domain. (There are no scalar factors since Theorem 2.12 implies that the coefficient of $L_{n}$ in the expansion of any $K_{P_{i}}(\mathbf{x})$ or $K_{Q_{j}}(\mathbf{x})$ in the $L_{\alpha}$-basis is 1 .) 
It is still open whether a connected poset $(P, \omega)$ that is not naturally labeled need have an irreducible generating function $K_{(P, \omega)}(\mathbf{x})$. Our approach will not work in this more general setting: for a general labeled poset $(P, \omega)$, it is possible for $K_{(P, \omega)}(\mathbf{x})$ to be irreducible even when $\widetilde{K}_{(P, \omega)}(\mathbf{x})$ is reducible. (Moreover, $K_{(P, \omega)}(\mathbf{x})$ may be irreducible yet lie in the span of homogeneous, reducible elements of QSym.)

Example 4.22. Let $(P, \omega)$ be the the following labeled poset with the generating function shown. (This can either be computed from the fundamental basis expansion using Theorem 2.12 and the results of [3], or from Theorem 6.9 below.)

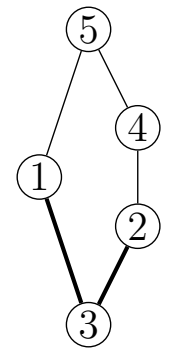

$$
\begin{aligned}
K_{(P, \omega)}(\mathbf{x})= & -\psi_{32}-\psi_{23}-\psi_{311}-\psi_{221}-3 \psi_{212}+\psi_{122} \\
& +\psi_{113}-3 \psi_{2111}+\psi_{1211}+\psi_{1121}+3 \psi_{1112}+3 \psi_{11111}
\end{aligned}
$$

The minimum length part of $K_{(P, \omega)}$ is then

$$
\begin{aligned}
\widetilde{K}_{(P, \omega)}(\mathbf{x}) & =-\psi_{32}-\psi_{23} \\
& =-\psi_{3} \cdot \psi_{2} .
\end{aligned}
$$

Since $\widetilde{K}_{(P, \omega)}(\mathbf{x})$ is reducible, it follows that $\tilde{\eta}\left(\widetilde{K}_{(P, \omega)}(\mathbf{x})\right)=0$ even though $P$ is connected. However, one can check that $K_{(P, \omega)}$ is itself irreducible (for instance, by considering the coefficients of $\psi_{311}, \psi_{131}$, and $\left.\psi_{113}\right)$.

\section{SERIES-PARALLEL POSETS}

We will now turn our attention to a collection of naturally labeled posets known as seriesparallel posets. We will use the results of the previous section to show that distinct seriesparallel posets have distinct partition generating functions.

Definition 5.1. The class $\mathcal{S P}$ of series-parallel posets is the smallest collection of posets that satisfies the following:

- the one-element poset $1_{\mathcal{P}}$ lies in $\mathcal{S P}$;

- if $P \in \mathcal{S P}$ and $Q \in \mathcal{S P}$, then $P \sqcup Q \in \mathcal{S P}$; and

- if $P \in \mathcal{S P}$ and $Q \in \mathcal{S P}$, then $P \oplus Q \in \mathcal{S P}$.

Here, the disjoint union $P \sqcup Q$ is the poset on the disjoint union of $P$ and $Q$ with relations $x \preceq y$ if and only if $x \preceq_{P} y$ or $x \preceq_{Q} y$, while the ordinal sum $P \oplus Q$ is the poset on the disjoint union of $P$ and $Q$ with relations $x \preceq y$ if and only if $x \preceq_{P} y, x \preceq_{Q} y$, or $x \in P$ and $y \in Q$.

It is well known that $P \in \mathcal{S P}$ if and only if $P$ is $N$-free [21]. (A poset is $N$-free if there does not exist an induced poset on four elements $\{a, b, c, d\} \subseteq P$ with relations $a \prec b \succ c \prec d$.) 
Note that if a series-parallel poset is disconnected, then it can be expressed as the disjoint union of series-parallel posets. If it is connected (and has more than one element), then it can be expressed as an ordinal sum of series-parallel posets.

In [8], Hasebe and Tsujie show that if a poset $P$ is a finite rooted tree, then it can be distinguished by its $P$-partition generating function. They then ask if the same can be said about series-parallel posets. We will now give an answer to this question.

Theorem 5.2. Let $P$ be a series-parallel poset. Then $P$ is uniquely determined (up to isomorphism) by $K_{P}(\mathbf{x})$.

Proof. We will prove this by induction on the size of $P$. If $P$ has a single element, then the result holds trivially since there is a unique single-element poset. Now suppose that the results holds for all series-parallel posets with fewer than $|P|$ elements.

If $P$ is disconnected, then $P=P_{1} \sqcup P_{2} \sqcup \cdots \sqcup P_{k}$, where each $P_{i}$ is a connected seriesparallel poset. By Lemma 4.20, the irreducible factors of $K_{P}(\mathbf{x})$ are the partition generating functions of the connected components of $P$. By induction, we can determine the connected components from their partition generating functions.

We will now assume that $P$ is connected and series-parallel. We can express $P$ as $P=$ $P_{1} \oplus P_{2} \oplus \cdots \oplus P_{k}$, where each $P_{i}$ is series-parallel. By Lemma 2.7 in [11], we can determine $K_{P_{i}}(\mathbf{x})$ from $K_{P}(\mathbf{x})$ for all $i$. Since each $P_{i}$ is series-parallel, it follows by induction that it is uniquely determined by $K_{P_{i}}(\mathbf{x})$.

It should be noted that Theorem 5.2 does not hold when $(P, \omega)$ is not naturally labeled.

Example 5.3. The following labeled series-parallel posets have the same partition generating functions.
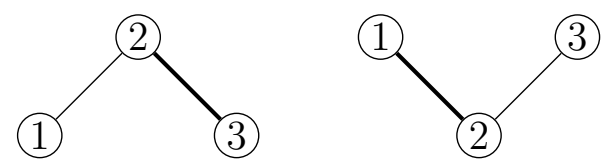

Although the labeled posets in Example 5.3 are not isomorphic, they are both seriesparallel.

Question 5.4. Let $\left(P, \omega_{1}\right)$ and $\left(Q, \omega_{2}\right)$ be labeled posets, and suppose that $P$ is seriesparallel. If $K_{\left(P, \omega_{1}\right)}(\mathbf{x})=K_{\left(Q, \omega_{2}\right)}(\mathbf{x})$, does this imply that $Q$ is series-parallel?

\section{A Combinatorial Description of the Coefficients}

In this section, we will give a combinatorial interpretation for the coefficients in the $\psi_{\alpha^{-}}$ expansion of $K_{(P, \omega)}(\mathbf{x})$ akin to the Murnaghan-Nakayama rule.

6.1. Generalized border-strips. We will begin by considering the following question: for which labeled posets $(P, \omega)$ does $\eta\left(K_{(P, \omega)}(\mathbf{x})\right) \neq 0$ ? By Lemma 3.3, this is equivalent to asking when the composition $(n)$ lies in the $\psi$-support of $K_{(P, \omega)}(\mathbf{x})$.

As mentioned earlier, in the fundamental quasisymmetric function basis $\left\{L_{\alpha}\right\}$,

$$
\eta\left(L_{\alpha}\right)= \begin{cases}(-1)^{k} & \text { if } \alpha=\left(1^{k}, n-k\right), \\ 0 & \text { otherwise }\end{cases}
$$

This means that we must only consider linear extensions of $(P, \omega)$ whose descent set is $\{1, \ldots, k\}$ for some value of $k$ (or empty when $k=0$ ) when computing $\eta\left(K_{(P, \omega)}(\mathbf{x})\right)$. 
Lemma 6.1. Suppose $(P, \omega)$ is a labeled poset. If there exists a chain $a \prec b \prec c$ such that $\omega(a)<\omega(b)>\omega(c)$, then $\eta\left(K_{(P, \omega)}(\mathbf{x})\right)=0$.

Proof. Every linear extension of $(P, \omega)$ has an ascent at some point between the appearance of $\omega(a)$ and $\omega(b)$, as well as a descent between the appearance of $\omega(b)$ and $\omega(c)$. Therefore $K_{(P, \omega)}(\mathbf{x})$ has no compositions of the form $\alpha=\left(1^{k}, n-k\right)$ in its $L$-support, so $\eta\left(K_{(P, \omega)}(\mathbf{x})\right)=$ 0 .

We say that a labeled poset $(P, \omega)$ is a generalized border-strip if $(P, \omega)$ does not contain a chain $a \prec b \prec c$ such that $\omega(a)<\omega(b)>\omega(c)$. Note that any generalized border-strip has a maximum order ideal $I$ containing only strict edges, in which case $P \backslash I$ is naturally labeled (i.e., contains only natural edges). The use of the term "generalized border-strip" will become clear in Section 6.3 when we consider labeled posets that arise from semistandard Young tableaux.

Denote by $J$ the maximal subset of $I$ such that $(P \backslash I) \cup J$ is naturally labeled and $I \backslash J$ is an order ideal. In other words, $J$ is the set of elements $j$ such that the principal order ideal generated by $j$ contains only strict edges, while the principal filter generated by $j$ contains only natural edges. Then $J$ must be a subset of the maximal elements of $I$. Note that for all $j \in J$ and $x \in P$, if $j$ and $x$ share an edge in the Hasse diagram of $(P, \omega)$, then $\omega(x)>\omega(j)$. Observe that $J$ is nonempty since the element labeled 1 (which we denote by 1 ) must be a maximal element in $I$, and $(P \backslash I) \cup\{1\}$ is naturally labeled, so $1 \in J$.

Example 6.2. Suppose that $(P, \omega)$ is the following generalized border-strip.

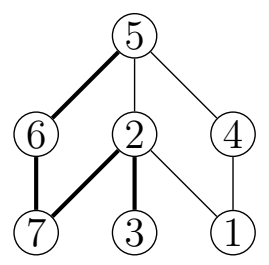

The ideal $I$ has maximal elements $\{1,3,6\}$, while $J=\{1\}$.

This set $J$ can be used to compute the value of $\eta\left(K_{(P, \omega)}(\mathbf{x})\right)$ for generalized border-strips.

Lemma 6.3. Suppose $(P, \omega)$ is a generalized border-strip, and let $I$ and $J$ be defined as above. Then

$$
\eta\left(K_{(P, \omega)}(\mathbf{x})\right)= \begin{cases}(-1)^{|I|-1} & \text { if } J=\{1\} \\ 0 & \text { otherwise. }\end{cases}
$$

Proof. We only need to consider the linear extensions of $(P, \omega)$ whose descent set is $\{1, \ldots, k\}$ for some value of $k$. The only linear extensions of $(P, \omega)$ with a descent set of this form must begin with, for some subset $A \subseteq J \backslash\{1\}$, the elements of $I \backslash A$ in decreasing order and end with the elements of $(P \backslash I) \cup A$ in increasing order. Therefore we can express $\eta\left(K_{(P, \omega)}(\mathbf{x})\right)$ as

$$
\eta\left(K_{(P, \omega)}(\mathbf{x})\right)=\sum_{A \subseteq J \backslash\{1\}}(-1)^{|I|-|A|-1} .
$$

This value is 0 unless $J=\{1\}$, in which case $\eta\left(K_{(P, \omega)}(\mathbf{x})\right)=(-1)^{|I|-1}$.

Observe that if $P$ is naturally labeled, then $\eta\left(K_{P}(\mathbf{x})\right)=1$ if and only if $P$ has a unique minimal element. (In this case, $I$ and $J$ are both just the set of minimal elements of $P$.) 
We say that $(P, \omega)$ is rooted if $\eta\left(K_{(P, \omega)}(\mathbf{x})\right) \neq 0$. In other words, $(P, \omega)$ is rooted if it is a generalized border-strip and $J=\{1\}$, that is, 1 is the unique element whose principal ideal contains only strict edges and whose principal filter contains only natural edges.

Corollary 6.4. If $(P, \omega)$ is rooted, then it is connected.

Proof. If $(P, \omega)$ is disconnected, then $K_{(P, \omega)}(\mathbf{x})$ is the product of the $(P, \omega)$-partition generating functions of the connected components of $P$. By Lemma 3.1. $\eta\left(K_{(P, \omega)}(\mathbf{x})\right)=0$.

Alternatively, the elements with the minimum label in each connected component of $P$ lie in the set $J$.

6.2. Enriched $(P, \omega)$-partitions. We will now give an alternate combinatorial description of rooted posets in terms of enriched $(P, \omega)$-partitions. We will then use this to give a combinatorial formula (with signs) for the coefficient of $\psi_{\alpha}$ in $K_{(P, \omega)}(\mathbf{x})$.

Let $\mathbf{P}^{\prime}$ be the nonzero integers with the total order

$$
-1<_{\mathbf{P}^{\prime}} 1<_{\mathbf{P}^{\prime}}-2<_{\mathbf{P}^{\prime}} 2<_{\mathbf{P}^{\prime}} \cdots .
$$

For $k \in \mathbf{P}^{\prime}$, we define the absolute value of $k$ by $|k|=i \in \mathbb{Z}^{+}$if $k \in\{-i, i\}$, and we say $k<0$ if $k=-|k|$, and $k>0$ otherwise.

The following definition is due to Stembridge [22].

Definition 6.5. Let $(P, \omega)$ be a labeled poset. An enriched $(P, \omega)$-partition is a map $f: P \rightarrow$ $\mathbf{P}^{\prime}$ such that $|f|$ is surjective onto $[k]$ for some $k$, and if $x$ is covered by $y$, then we have

(i) $f(x) \leq_{\mathbf{P}^{\prime}} f(y)$,

(ii) if $|f(x)|=|f(y)|$ and $\omega(x)<\omega(y)$, then $f(y)>0$,

(iii) if $|f(x)|=|f(y)|$ and $\omega(x)>\omega(y)$, then $f(x)<0$.

It should be noted that if $x \prec y \prec z$ is a chain with $\omega(x)<\omega(y)>\omega(z)$, then for all enriched $(P, \omega)$-partitions $f,|f(x)|<|f(z)|$. It follows that for each $i$, the subposet $P_{i}=\{x \in P|| f(x) \mid=i\}$ is a generalized border-strip.

Suppose that $f$ is an enriched $(P, \omega)$-partition and we know the value of $|f(x)|$ for all $x$. Is this enough information to determine the value of $f(x)$ for all $x$ ? The answer to this question is no.

Example 6.6. Let $(P, \omega)$ be the following poset. Suppose $f$ is an enriched $(P, \omega)$-partition, and $|f(x)|=1$ for all $x$.

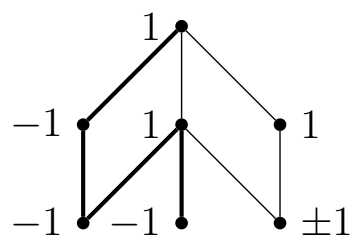

The element in the bottom right can either be sent to -1 or 1 by $f$.

For each $x \in P$ and enriched $(P, \omega)$-partition $f$, define the map $f_{x}: P \rightarrow \mathbf{P}^{\prime}$ by

$$
f_{x}(y)= \begin{cases}-f(x) & \text { if } x=y \\ f(y) & \text { otherwise }\end{cases}
$$

We say that an element $x \in P$ is ambiguous with respect to $f$ if $f_{x}$ is still an enriched $(P, \omega)$-partition. 
Let $\mathbf{P}^{*}=\mathbf{P}^{\prime} \cup\left\{1^{*}, 2^{*}, \ldots\right\}$, where each $i^{*}$ satisfies $-i<\mathbf{P}^{*} i^{*}<_{\mathbf{P}^{*}} i$ and $\left|i^{*}\right|=i$. For an enriched $(P, \omega)$-partition $f$, consider the map $f^{*}: P \rightarrow \mathbf{P}^{*}$ defined by

$$
f^{*}(x)= \begin{cases}|f(x)|^{*} & \text { if } x \text { is ambiguous with respect to } f \\ f(x) & \text { otherwise. }\end{cases}
$$

The map $f^{*}$ is called a starred $(P, \omega)$-partition. Note that $f^{*}$ is uniquely determined by the generalized border-strips $P_{i}$.

The ambiguity of $f^{*}$ is the sequence $\operatorname{amb}\left(f^{*}\right)=\left(a_{1}, a_{2}, \ldots, a_{k}\right)$, where $a_{i}=\left|\left(f^{*}\right)^{-1}\left(i^{*}\right)\right|$, the number of elements labeled $i^{*}$ by $f^{*}$, The sign of $f^{*}$ is

$$
\operatorname{sign}\left(f^{*}\right)=(-1)^{\left|\left\{x \mid f^{*}(x)<0\right\}\right|},
$$

and the weight of $f^{*}$ is $\operatorname{wt}\left(f^{*}\right)=\left(b_{1}, b_{2}, \ldots, b_{k}\right)$, where $b_{i}$ is the total number of elements labeled $-i, i^{*}$, or $i$ by $f$.

Example 6.7. Let $f$ be the enriched $(P, \omega)$-partition from Example 6.6. Then the starred $(P, \omega)$-partition $f^{*}$ is shown below.

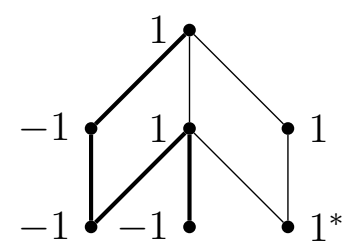

Here, $\operatorname{amb}\left(f^{*}\right)=(1), \operatorname{sign}\left(f^{*}\right)=(-1)^{3}$, and $\operatorname{wt}\left(f^{*}\right)=(7)$.

We say that a starred $(P, \omega)$-partition $f^{*}$ is a pointed $(P, \omega)$-partition if $\operatorname{amb}\left(f^{*}\right)=$ $(1,1, \ldots, 1)$. For instance, if $f: P \rightarrow[n]$ is any linear extension of $(P, \omega)$, then $f^{*}$ is a pointed $(P, \omega)$-partition. When $P$ is naturally labeled, $f^{*}$ will be pointed whenever each subposet $P_{i}=\left\{x \in P|| f^{*}(x) \mid=i\right\}$ has a unique minimal element, so this agrees with our previous definition of pointed $P$-partitions.

The following lemma is equivalent to Lemma 6.3

Lemma 6.8. Let $(P, \omega)$ be a labeled poset. Then there exists a pointed $(P, \omega)$-partition $f^{*}$ with $\operatorname{wt}\left(f^{*}\right)=(n)$ if and only if $(P, \omega)$ is rooted. In this case, $\eta\left(K_{(P, \omega)}(\mathbf{x})\right)=\operatorname{sign}\left(f^{*}\right)$; otherwise $\eta\left(K_{(P, \omega)}(\mathbf{x})\right)=0$.

Proof. We know from Lemma 6.1 that in order for $\eta\left(K_{(P, \omega)}(\mathbf{x})\right) \neq 0$, there cannot be a chain $a \prec b \prec c$ with $\omega(a)<\omega(b)>\omega(c)$. If there are no such chains, then there exists a starred $(P, \omega)$-partition $f^{*}$ with $\operatorname{wt}\left(f^{*}\right)=(n)$. Explicitly, $f^{*}$ labels the elements of $P$ is as follows: if an element lies at the bottom of a strict edge, then it gets sent to -1 ; if it lies at the top of a natural edge, then it gets sent to 1 ; otherwise, it gets sent to $1^{*}$. There are no elements that lie both at the bottom of a strict edge and at the top of a natural edge.

The elements that get sent to $1^{*}$ are what we called $J$ in Lemma 6.3, and the elements that get sent to -1 or $1^{*}$ are what we called $I$. Therefore the result holds by Lemma 6.3.

A pointed $(P, \omega)$-partition $f^{*}$ can be interpreted as a partitioning of $(P, \omega)$ such that each part is rooted. Specifically, each subposet $P_{i}=\left\{x \in P|| f^{*}(x) \mid=i\right\}$ is a rooted poset, and the weight of $f^{*}$ is the composition $\left(\left|P_{1}\right|,\left|P_{2}\right|, \ldots\right)$. This allows us to give a combinatorial description of the coefficient of $\psi_{\alpha}$ in $K_{(P, \omega)}(\mathbf{x})$. 
Theorem 6.9. Let $(P, \omega)$ be a labeled poset. Then

$$
K_{(P, \omega)}(\mathbf{x})=\sum_{f^{*}} \operatorname{sign}\left(f^{*}\right) \psi_{\mathrm{wt}\left(f^{*}\right)}
$$

where the sum ranges over all pointed $(P, \omega)$-partitions $f^{*}$.

Proof. Recall that Theorem 3.5 states that the coefficient of $\psi_{\alpha}$ in $K_{(P, \omega)}(\mathbf{x})$ can be computed by $\eta^{\otimes l}\left(\Delta_{\alpha} K_{(P, \omega)}(\mathbf{x})\right)$, and that

$$
\Delta_{\alpha} K_{(P, \omega)}(\mathbf{x})=\sum K_{\left(P_{1}, \omega\right)}(\mathbf{x}) \otimes \cdots \otimes K_{\left(P_{l}, \omega\right)}(\mathbf{x})
$$

where $\left|P_{i}\right|=\alpha_{i}, P_{1} \cup \cdots \cup P_{i}$ is an order ideal of $P$, and $P_{1}, P_{2}, \ldots, P_{l(\alpha)}$ partition $P$.

Therefore the coefficient of $\psi_{\alpha}$ in $K_{(P, \omega)}(\mathbf{x})$ is given by

$$
\eta^{\otimes l}\left(\Delta_{\alpha} K_{(P, \omega)}(\mathbf{x})\right)=\sum \eta\left(K_{\left(P_{1}, \omega\right)}(\mathbf{x})\right) \otimes \cdots \otimes \eta\left(K_{\left(P_{l}, \omega\right)}(\mathbf{x})\right) .
$$

By Lemma 6.8, a term in this summation is 0 unless each $\left(P_{i}, \omega\right)$ is rooted, meaning there exists a pointed $\left(P_{i}, \omega\right)$-partition $f_{i}^{*}$ with weight $\left(\alpha_{i}\right)$. These can be combined to form a unique pointed $(P, \omega)$-partition $f^{*}$ with $\operatorname{wt}\left(f^{*}\right)=\alpha$. Since $\eta\left(K_{\left(P_{i}, \omega\right)}(\mathbf{x})\right)=\operatorname{sign}\left(f_{i}^{*}\right)$, it follows that $\eta\left(K_{P_{1}}(\mathbf{x})\right) \otimes \cdots \otimes \eta\left(K_{P_{l}}(\mathbf{x})\right)=\operatorname{sign}\left(f^{*}\right)$.

Example 6.10. Let $(P, \omega)$ be the following poset.

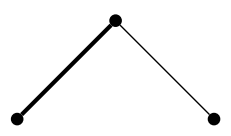

The following are the pointed $(P, \omega)$-partitions.

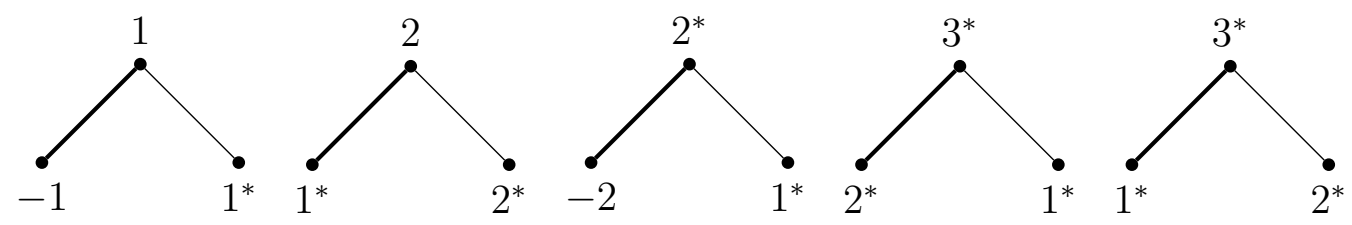

Therefore $K_{(P, \omega)}(\mathbf{x})=-\psi_{3}+\psi_{12}-\psi_{12}+2 \psi_{111}=-\psi_{3}+2 \psi_{111}$.

Observe that although the labeled poset in the previous example has a pointed $(P, \omega)$ partition with weight $(1,2)$, the composition $(1,2)$ is not in the $\psi$-support of $K_{(P, \omega)}(\mathbf{x})$.

Question 6.11. What is the $\psi$-support of $K_{(P, \omega)}(\mathbf{x})$ ?

One should note that the combinatorial interpretation given in Theorem 6.9 is consistent with the description given in [2] (or Corollary 3.7 above) when $(P, \omega)$ in naturally labeled.

6.3. Murnaghan-Nakayama rule. In this section, we will compare Theorem 6.9 to the Murnaghan-Nakayama rule, which expresses a Schur symmetric function in terms of power sum symmetric functions.

We begin with some background on symmetric functions and tableaux. (For more information, see [16, 19].) A symmetric function in the variables $x_{1}, x_{2}, \ldots$ (with coefficients in $\mathbb{C})$ is a formal power series $f(\mathbf{x}) \in \mathbb{C}[[\mathbf{x}]]$ of bounded degree such that, for any composition $\alpha$, the coefficient of $x_{1}^{\alpha_{1}} x_{2}^{\alpha_{2}} \cdots x_{k}^{\alpha_{k}}$ equals the coefficient of $x_{i_{1}}^{\alpha_{1}} x_{i_{2}}^{\alpha_{2}} \cdots x_{i_{k}}^{\alpha_{k}}$ whenever $i_{1}, i_{2}, \ldots, i_{k}$ are distinct. We denote the algebra of symmetric functions by $\Lambda$. Clearly every symmetric function is also quasisymmetric. 
The power sum symmetric function basis $\left\{p_{\lambda}\right\}$, indexed by partitions $\lambda$, is given by $p_{n}=$ $x_{1}^{n}+x_{2}^{n}+\cdots$ and $p_{\lambda}=p_{\lambda_{1}} p_{\lambda_{2}} \cdots$. In [3], it was shown that

$$
\frac{p_{\lambda}}{z_{\lambda}}=\sum_{\alpha \sim \lambda} \psi_{\alpha}
$$

where the sum runs over all compositions $\alpha$ that rearrange to the partition $\lambda$.

For any partition $\lambda$, the Young diagram of shape $\lambda$ is a collection of boxes arranged in left-justified rows such that row $i$ has $\lambda_{i}$ boxes. If $\lambda$ and $\mu$ are partitions such that $\mu_{i} \leq \lambda_{i}$ for all $i$, then the Young diagram of the (skew) shape $\lambda / \mu$ is the set-theoretic difference between the Young diagram of shape $\lambda$ and the Young diagram of shape $\mu$.

A semistandard (Young) tableau (SSYT) of (skew) shape $\lambda / \mu$ is a labeling of the boxes of the Young diagram of shape $\lambda / \mu$ such that the entries in the rows are weakly increasing from left to right, and the entries in the columns are strictly increasing from top to bottom. If $T$ is an SSYT of shape $\lambda / \mu$, then we write $\lambda / \mu=\operatorname{sh}(T)$. We say that $T$ has type $\alpha=\left(\alpha_{1}, \alpha_{2}, \ldots\right)$, denoted $\alpha=\operatorname{type}(T)$, if $T$ has $\alpha_{i}=\alpha_{i}(T)$ parts equal to $i$. For any SSYT $T$ of type $\alpha$, we write $x^{T}=x_{1}^{\alpha_{1}(T)} x_{2}^{\alpha_{2}(T)} \ldots$. The skew Schur function $s_{\lambda / \mu}$ is the formal power series

$$
s_{\lambda / \mu}(\mathbf{x})=\sum_{T} x^{T}
$$

where the sum runs over all SSYT $T$ of shape $\lambda / \mu$. The Schur functions $\left\{s_{\lambda}\right\}$ for partitions $\lambda$ form a basis for $\Lambda$.

Example 6.12. The following is an SSYT of shape $652 / 21$ and type $(3,3,3,1)$.

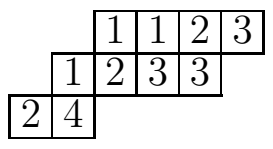

Define $P_{\lambda / \mu}$ to be the poset whose elements are the squares $(i, j)$ of $\lambda / \mu$, partially ordered componentwise. Define a labeling $\omega_{\lambda / \mu}: P_{\lambda / \mu} \rightarrow[n]$ as follows: first, the bottom square of the first column of $P_{\lambda / \mu}$ is labeled 1. The labeling then proceeds in order up the first column, then up the second column, and so forth.

Example 6.13. The following is $\left(P_{\lambda / \mu}, \omega_{\lambda / \mu}\right)$ when $\lambda=652$ and $\mu=21$.

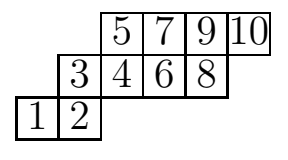

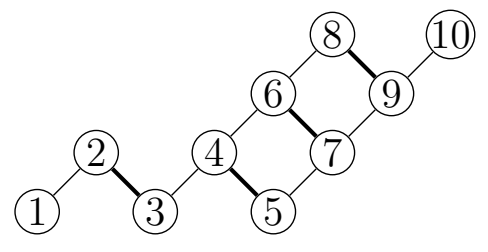

It follows immediately from the definition of $K_{(P, \omega)}(\mathbf{x})$ that

$$
K_{\left(P_{\lambda / \mu}, \omega_{\lambda / \mu}\right)}(\mathbf{x})=\sum_{T} x^{T}=s_{\lambda / \mu}(\mathbf{x})
$$

where the sum runs over all SSYT $T$ of shape $\lambda / \mu$.

We can express $s_{\lambda / \mu}$ in terms of the power sum symmetric function basis using the following combinatorial rule, known as the Murnaghan-Nakayama rule.

A border strip is a connected skew shape with no $2 \times 2$ square. Define the height ht $(B)$ of a border strip $B$ to be one less than its number of rows. 
A border-strip tableau of shape $\lambda / \mu$ and type $\alpha=\left(\alpha_{1}, \alpha_{2}, \ldots\right)$ is an assignment of positive integers to the squares of $\lambda / \mu$ such that

- every row and column is weakly increasing,

- the integer $i$ appears $\alpha_{i}$ times, and

- the set of squares occupied by $i$ forms a border strip.

The height of a border-strip tableau $T$, denoted ht $(T)$, is the sum of the heights of the border strips that make up $T$.

Theorem 6.14 (Murnaghan-Nakayama rule). For partitions $\lambda, \mu$, and $\nu$,

$$
s_{\lambda / \mu}=\sum_{\nu} \chi^{\lambda / \mu}(\nu) \frac{p_{\nu}}{z_{\nu}}
$$

Here $\chi^{\lambda / \mu}(\nu)=\sum_{T}(-1)^{\mathrm{ht}(T)}$, where the sum ranges over all border-strip tableaux of shape $\lambda / \mu$ and type $\nu$.

When $\mu=\varnothing$, Theorem 6.14 gives a change of basis formula for expressing Schur functions in terms of the power sum symmetric functions.

Since the quasisymmetric power sums refine the symmetric power sums, the MurnaghanNakayama rule also gives a description of the $\psi_{\alpha}$-expansion of $s_{\lambda / \mu}$. Since $\chi^{\lambda / \mu}(\nu)$ also does not depend on the order of the parts of $\nu$ [19], we find that the Murnaghan-Nakayama rule is equivalent to

$$
s_{\lambda / \mu}=\sum_{\alpha} \chi^{\lambda / \mu}(\alpha) \psi_{\alpha}
$$

where the sum ranges over all compositions $\alpha$.

In fact, this description agrees with the one obtained by Theorem 6.9 above as we will next demonstrate.

Proposition 6.15. When $(P, \omega)=\left(P_{\lambda / \mu}, \omega_{\lambda / \mu}\right), \eta\left(K_{(P, \omega)}(\mathbf{x})\right)=0$ unless $\lambda / \mu$ is a border strip, in which case $\eta\left(K_{(P, \omega)}(\mathbf{x})\right)=(-1)^{\mathrm{ht}(\lambda / \mu)}$.

Proof. If $\lambda / \mu$ contains a $2 \times 2$ square, then $\left(P_{\lambda / \mu}, \omega_{\lambda / \mu}\right)$ contains a chain $a \prec b \prec c$ with $\omega(a)<\omega(b)>\omega(c)$. It follows from Lemma 6.1 that $\eta\left(K_{(P, \omega)}(\mathbf{x})\right)=0$. Similarly, if $\left(P_{\lambda / \mu}, \omega_{\lambda / \mu}\right)$ is disconnected, then by Corollary 6.4, we have $\eta\left(K_{(P, \omega)}(\mathbf{x})\right)=0$. Therefore the only way to have $\eta\left(K_{(P, \omega)}(\mathbf{x})\right) \neq 0$ is if $\lambda / \mu$ is connected and contains no $2 \times 2$ square, that is, if it is a border strip.

Using the terminology of Lemma 6.3, when $\lambda / \mu$ is a border strip, the elements of $\left(P_{\lambda / \mu}, \omega_{\lambda / \mu}\right)$ that lie in $I$ are the leftmost boxes in the rows of the Young diagram with shape $\lambda / \mu$. Therefore $|I|$ is equal to the number of rows of $\lambda / \mu$. The only element of $J$ is the box in the southwest corner. By Lemma 6.3, we have that $\eta\left(K_{(P, \omega)}(\mathbf{x})\right)=(-1)^{\mathrm{ht}(\lambda / \mu)}$.

Example 6.16. The following is $\left(P_{\lambda / \mu}, \omega_{\lambda / \mu}\right)$ when $\lambda=6332$ and $\mu=221$.
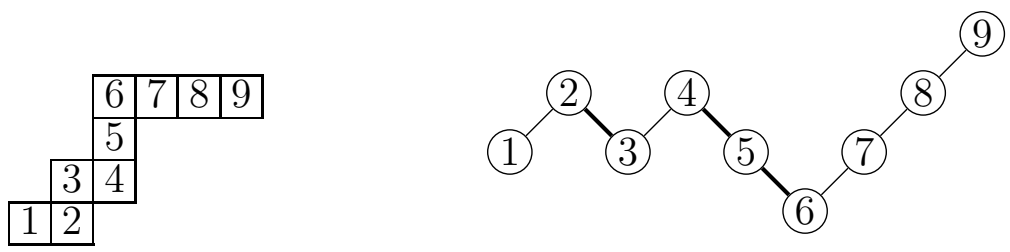

Since $\lambda / \mu$ is a border strip, it follows that $\eta\left(K_{\left(P_{\lambda / \mu}, \omega_{\lambda / \mu}\right)}(\mathbf{x})\right)=(-1)^{3}$. We see that $I=$ $\{1,3,5,6\}$ and $J=\{1\}$. 
Corollary 6.17. For partitions $\lambda$ and $\mu$ and compositions $\alpha$,

$$
s_{\lambda / \mu}=\sum_{\alpha} \chi^{\lambda / \mu}(\alpha) \psi_{\alpha}
$$

Here $\chi^{\lambda / \mu}(\alpha)=\sum_{T}(-1)^{\mathrm{ht}(T)}$, where the sum ranges over all border-strip tableaux of shape $\lambda / \mu$ and type $\alpha$.

Proof. By Proposition 6.15, a pointed $\left(P_{\lambda / \mu}, \omega_{\lambda / \mu}\right)$-partition $f^{*}$ is equivalent to a border-strip tableau $T$ of shape $\lambda / \mu$. In this case, $\operatorname{wt}\left(f^{*}\right)=\operatorname{type}(T)$ and $\operatorname{sign}\left(f^{*}\right)=(-1)^{\mathrm{ht}(T)}$, so the result follows immediately from Theorem 6.9.

Example 6.18. Let $\lambda=(6,4,4,4,2)$ and $\mu=(3,2,1,1)$. The following is a border strip tableau of shape $\lambda / \mu$ and the pointed $\left(P_{\lambda / \mu}, \omega_{\lambda / \mu}\right)$-partition $f^{*}$ that corresponds to it.
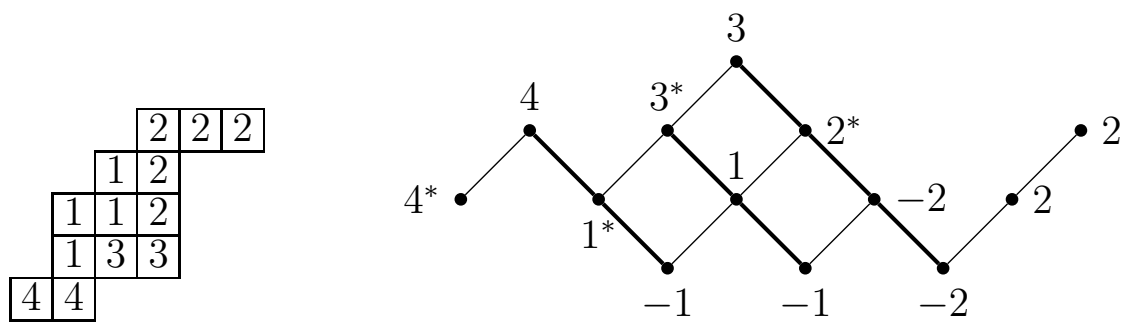

\section{ACKNOWLEDGMENTS}

The authors would like to thank Peter McNamara and Robin Sulzgruber for useful conversations.

\section{REFERENCES}

[1] Marcelo Aguiar, Nantel Bergeron, and Frank Sottile. Combinatorial Hopf algebras and generalized Dehn-Sommerville relations. Compos. Math., 142(1):1-30, 2006.

[2] Per Alexandersson and Robin Sulzgruber. P-partitions and p-positivity. International Mathematics Research Notices. To appear.

[3] Cristina Ballantine, Zajj Daugherty, Angela Hicks, Sarah Mason, and Elizabeth Niese. Quasisymmetric Power Sums. arXiv e-prints, October 2017. Available at arXiv:1710.11613.

[4] Louis J. Billera, Hugh Thomas, and Stephanie van Willigenburg. Decomposable compositions, symmetric quasisymmetric functions and equality of ribbon Schur functions. Adv. Math., 204(1):204-240, 2006.

[5] Israel M. Gelfand, Daniel Krob, Alain Lascoux, Bernard Leclerc, Vladimir S. Retakh, and Jean-Yves Thibon. Noncommutative symmetric functions. Adv. Math., 112(2):218-348, 1995.

[6] Ira Gessel. Multipartite $P$-partitions and inner products of skew schur functions. Contemporary Mathematics, 34:289-301, 1984.

[7] Darij Grinberg and Victor Reiner. Hopf Algebras in Combinatorics. arXiv e-prints, Sep 2014. Available at arXiv:1409.8356.

[8] Takahiro Hasebe and Shuhei Tsujie. Order quasisymmetric functions distinguish rooted trees. Journal of Algebraic Combinatorics, 46(3-4):499-515, 2017.

[9] Michiel Hazewinkel. The algebra of quasi-symmetric functions is free over the integers. Adv. Math., 164(2):283-300, 2001.

[10] Thomas Lam and Pavlo Pylyavskyy. $P$-partition products and fundamental quasi-symmetric function positivity. Adv. in Appl. Math., 40(3):271-294, 2008.

[11] Ricky Ini Liu and Michael Weselcouch. $P$-partition generating function equivalence of naturally labeled posets. J. Comb. Theory Ser. A, 170:105136, 2020. 
[12] Kurt Luoto, Stefan Mykytiuk, and Stephanie van Willigenburg. An introduction to quasisymmetric Schur functions. SpringerBriefs in Mathematics. Springer, New York, 2013. Hopf algebras, quasisymmetric functions, and Young composition tableaux.

[13] Peter R. W. McNamara and Stephanie van Willigenburg. Towards a combinatorial classification of skew Schur functions. Trans. Amer. Math. Soc., 361(8):4437-4470, 2009.

[14] Peter R. W. McNamara and Ryan E. Ward. Equality of P-partition generating functions. Annals of Combinatorics, 18(3):489-514, 2014.

[15] Victor Reiner, Kristen M. Shaw, and Stephanie van Willigenburg. Coincidences among skew Schur functions. Adv. Math., 216(1):118-152, 2007.

[16] Bruce E. Sagan. The symmetric group, volume 203 of Graduate Texts in Mathematics. Springer-Verlag, New York, second edition, 2001. Representations, combinatorial algorithms, and symmetric functions.

[17] William R. Schmitt. Incidence Hopf algebras. J. Pure Appl. Algebra, 96(3):299-330, 1994.

[18] Richard P. Stanley. Ordered structures and partitions. American Mathematical Society, Providence, R.I., 1972. Memoirs of the American Mathematical Society, No. 119.

[19] Richard P. Stanley. Enumerative combinatorics. Vol. 2, volume 62 of Cambridge Studies in Advanced Mathematics. Cambridge University Press, Cambridge, 1999. With a foreword by Gian-Carlo Rota and appendix 1 by Sergey Fomin.

[20] Richard P. Stanley. The descent set and connectivity set of a permutation. J. Integer Seq., 8(3):Article 05.3.8, 9, 2005.

[21] Richard P. Stanley. Enumerative combinatorics. Vol. 1, volume 49 of Cambridge Studies in Advanced Mathematics. Cambridge University Press, Cambridge, second edition, 2012.

[22] John R. Stembridge. Enriched P-partitions. Trans. Amer. Math. Soc., 349(2):763-788, 1997.

Department of Mathematics, North Carolina State University, Raleigh, NC

E-mail address: riliu@ncsu.edu

Department of Mathematics, North Carolina State University, Raleigh, NC

E-mail address: mweselc@ncsu.edu 